\title{
LIE ALGEBRAS OF VECTOR FIELDS IN THE REAL PLANE
}

\author{
ARTEMIO GONZÁLEZ-LÓPEZ, NIKY KAMRAN, \\ and PETER J. OLVER
}

[Received 9 March 1990-Revised 28 November 1990]

\begin{abstract}
Finite-dimensional real analytic Lie algebras of vector fields on $\mathbb{R}^{2}$ are completely classified up to changes of local coordinates.
\end{abstract}

\section{Introduction}

In the early days of Lie theory, the problem of classifying Lie algebras of vector fields under local diffeomorphisms played a central role in the subject, notably because of the applications to the integration of differential equations admitting infinitesimal symmetries. Lie himself classified the Lie algebras of vector fields in one real variable, one complex variable and two complex variables (see Lie [14, 15], Bianchi [2], Campbell [5], Hermann and Ackermann [9]). He also outlined an ingenious geometric argument which enabled him to list the Lie algebras of vector fields in two real variables [16, p. 360]. Finally, in [16, pp. 122-178], Lie claimed to have completely classified all Lie algebras of vector fields in three complex variables, but writes that it would take too much space to present the complete results. Instead, he gives details in the case of primitive algebras, and divides the imprimitive cases into three classes, of which only the first two are treated. It is not known whether the remaining calculations still exist. The prohibitive complexity of the calculations required to classify the Lie algebras of vector fields under local diffeomorphisms in more than two variables and the new directions opened by Élie Cartan's classification of abstract semisimple complex Lie algebras [6], led the researchers working in Lie theory to turn their attention to more algebraic problems, such as the ones arising in the representation theory of Lie algebras.

Recently, there has been a revival of interest in the local classification problem for Lie algebras of vector fields, owing notably to their role in geometric control theory [4], in the theory of systems of non-linear ordinary differential equations with superposition principles [21, 23], and in the so-called algebraic approach to molecular dynamics [1]. It is this latter connection which motivates the present work. Specifically, R. Levine [13] posed the problem of classifying all the second-order time independent Schrödinger operators which are elements of the universal enveloping algebra of a finite-dimensional Lie algebra $g$ of first-order differential operators. The solution of Levine's problem requires as a first step the classification of the Lie algebras of vector fields. In a pair of recent papers $[11,12]$ two of the present authors gave a complete solution to Levine's problem in the

The research of the second author was partially supported by an NSERC grant, and that of the third author by NSF Grant DMS 89-01600.

1991 Mathematics Subject Classification: 17B66, 57S25.

Proc. London Math. Soc. (3) 64 (1992) 339-368. 
one-dimensional case, that is for Schrödinger operators on the real or complex line. The classification of the Lie algebras of first-order differential operators in two complex variables, based on Lie's classification of Lie algebras of vector fields in two complex variables, was also completed recently [8]. In the present paper, we carry out the first step in the solution of Levine's problem in the two-dimensional real case by performing a complete classification of the Lie algebras of vector fields in two real variables. There are twenty-eight equivalence classes (some of them depending on essential parameters), for which we present a complete list of normal forms. (As with Lie's classification, this classification holds at 'generic points' away from singularities.) While the algebras we obtain correspond to those listed earlier by Lie, we are not aware of any existing attempt to do the classification using rigorous methods from the modern theory of Lie algebras. We will apply these results to the classification of Lie algebras of differential operators in two real variables and the solution to Levine's problem in subsequent publications.

The classification proceeds according to whether the Lie algebra of vector fields considered is primitive or imprimitive. In the imprimitive case, the classification is the same in the real and complex cases, and thus gives rise to twenty parametrized equivalence classes. (This is also treated in Chapter E of [9].) The major part of our paper is thus devoted to the case of primitive Lie algebras of vector fields, for which the results are considerably more complicated in the real than in the complex case. Indeed, in addition to the three equivalence classes obtained by Lie in the complex case, we obtain five new equivalence classes of Lie algebras. These Lie algebras, for which we present normal forms, are equivalent upon analytic continuation to one of the previous ones under a complex local diffeomorphism, but not under a real local diffeomorphism. The complete classification and list of normal forms for all finite-dimensional Lie algebras of vector fields in $\mathbb{R}^{2}$ (our 'Real Gruppenregister', being the counterpart of Lie's Complex Gruppenregister given in reference [14]) are conveniently collected together in Table 1 (see $\S 9$ for a detailed explanation).

These results will, we hope, clear up some confusing and contradictory statements in the literature on this problem. The classical authors, for example, Lie, Campbell, Bianchi, etc., never really made it clear whether they were working over the real or the complex numbers, which has led to confusion among more recent authors. For example, in their translation and commentary on Lie's paper [9], Hermann and Ackerman assert that the lemma on triangularization of matrices holds only over the complex numbers [9, p. 296], while the commentary is clearly aimed at real vector fields. A similar mis-statement occurs in Bluman and Kumei's recent book on symmetry groups of differential equations [3, p. 129], where it is falsely asserted that Campbell [5] obtained the classification of vector fields over $\mathbb{R}^{2}$. Bluman and Kumei go on to use Campbell's complex classification to state a theorem which does not hold in the real case: that every Lie algebra of vector fields on the plane contains a two-dimensional subalgebra, a result which has important consequences for the integrability of ordinary differential equations using symmetry groups. Interestingly, the only counter-example to Bluman and Kumei's assertion is the Lie algebra $\mathfrak{s} \mathfrak{v}(3, \mathbb{R})$, which has an action on $\mathbb{R}^{2}$, obtained by stereographically projecting its standard action by infinitesimal rotations on the unit sphere $S^{2}$; see $\S 7$, and entry 3 in Table 1 . 
TABLE 1. Finite-dimensional Lie algebras of vector fields in the real plane

I. Locally primitive algebras

\begin{tabular}{|c|c|}
\hline Generators & Structure \\
\hline 1. $\{p, q, \alpha(x p+y q)+y p-x q\}, \alpha \geqslant 0$ & $\mathbb{R} \ltimes \mathbb{R}^{2}$ \\
\hline 2. $\left\{p, x p+y q,\left(x^{2}-y^{2}\right) p+2 x y q\right\}$ & $\mathfrak{\xi l}(2)$ \\
\hline 3. $\left\{y p-x q,\left(1+x^{2}-y^{2}\right) p+2 x y q, 2 x y p+\left(1+y^{2}-x^{2}\right) q\right\}$ & $\mathfrak{s o}(3)$ \\
\hline 4. $\{p, q, x p+y q, y p-x q\}$ & $\mathbb{P}^{2} \ltimes \mathbb{R}^{2}$ \\
\hline 5. $\{p, q, x p-y q, y p, x q\}$ & $\mathfrak{s l}(2) \ltimes \mathbb{R}^{2}$ \\
\hline 6. $\{p, q, x p, y p, x q, y q\}$ & $\mathfrak{g l}(2) \ltimes \mathbb{R}^{2}$ \\
\hline $\begin{array}{l}\text { 7. }\left\{p, q, x p+y q, y p-x q,\left(x^{2}-y^{2}\right) p+2 x y q\right. \\
\left.2 x y p+\left(y^{2}-x^{2}\right) q\right\}\end{array}$ & $\mathfrak{s o}(3,1)$ \\
\hline 8. $\left\{p, q, x p, y p, x q, y q, x^{2} p+x y q, x y p+y^{2} q\right\}$ & $\mathfrak{S I}(3)$ \\
\hline
\end{tabular}

II. Imprimitive algebras

\begin{tabular}{|c|c|}
\hline Generators & Structure \\
\hline 9. $\{p\}$ & $\mathbb{R}$ \\
\hline 10. $\{p, x p\}$ & $\mathfrak{h}_{2}$ \\
\hline 11. $\left\{p, x p, x^{2} p\right\}$ & $\mathfrak{S I}(2)$ \\
\hline 12. $\{p, q, x p+\alpha y q\}, 0<|\alpha| \leqslant 1$ & $\mathbb{B} \ltimes \mathbb{R}^{2}$ \\
\hline 13. $\{p, q, x p, y q\}$ & $\mathfrak{h}_{2} \oplus \mathfrak{h}_{2}$ \\
\hline 14. $\left\{p, q, x p, x^{2} p\right\}$ & $\operatorname{gl}(2)$ \\
\hline 15. $\left\{p, q, x p, y q, x^{2} p\right\}$ & $\mathfrak{G l}(2) \oplus \mathfrak{h}_{2}$ \\
\hline 16. $\left\{p, q, x p, y q, x^{2} p, y^{2} q\right\}$ & $\mathfrak{g l}(2) \oplus \mathfrak{s l}(2) \approx \mathfrak{s} \mathfrak{v}(2,2)$ \\
\hline 17. $\left\{p+q, x p+y q, x^{2} p+y^{2} q\right\}$ & $\operatorname{sI}(2)$ \\
\hline 18. $\left\{p, 2 x p+y q, x^{2} p+x y q\right\}$ & $\mathfrak{s l}(2)$ \\
\hline 19. $\left\{p, x p, y q, x^{2} p+x y q\right\}$ & $\operatorname{gl}(2)$ \\
\hline 20. $\left\{q, \xi_{1}(x) q, \ldots, \xi_{r}(x) q\right\}, r \geqslant 1$ & $\mathbb{R}^{r+1}$ \\
\hline 21. $\left\{q, y q, \xi_{1}(x) q, \ldots, \xi_{r}(x) q\right\}, r \geqslant 1$ & $\mathbb{R} \ltimes \mathbb{R}^{r+1}$ \\
\hline 22. $\left\{p, \eta_{1}(x) q, \ldots, \eta_{r}(x) q\right\}, r \geqslant 1$ & $\mathbb{R} \ltimes \mathbb{R}^{r}$ \\
\hline 23. $\left\{p, y q, \eta_{1}(x) q, \ldots, \eta_{r}(x) q\right\}, r \geqslant 1$ & $\mathbb{R}^{2} \ltimes \mathbb{R}^{r}$ \\
\hline 24. $\left\{p, q, x p+\alpha y q, x q, \ldots, x^{r} q\right\}, r \geqslant 1$ & $\mathfrak{h}_{2} \ltimes \mathbb{R}^{r+1}$ \\
\hline 25. $\left\{p, q, x q, \ldots, x^{r-1} q, x p+\left(r y+x^{r}\right) q\right\}, r \geqslant 1$ & $\mathbb{R} \ltimes\left(\mathbb{R} \propto \mathbb{R}^{r}\right)$ \\
\hline 26. $\left\{p, q, x p, x q, y q, x^{2} q, \ldots, x^{r} q\right\}, r \geqslant 1$ & $\left(\mathfrak{h}_{2} \oplus \mathbb{R}\right) \ltimes \mathbb{R}^{r+1}$ \\
\hline 27. $\left\{p, q, 2 x p+r y q, x q, x^{2} p+r x y q, x^{2} q \ldots, x^{r} q\right\}, r \geqslant 1$ & $\mathfrak{S I}(2) \propto \mathbb{R}^{r+1}$ \\
\hline 28. $\left\{p, q, x p, x q, y q, x^{2} p+r x y q, x^{2} q \ldots, x^{r} q\right\}, r \geqslant 1$ & $\mathfrak{g l}(2) \ltimes \mathbb{R}^{r+1}$ \\
\hline
\end{tabular}

\section{Preliminaries}

We shall collect in the following section a few definitions and basic results which will be useful in the rest of the paper. In what follows, $g$ will denote a real analytic Lie algebra of vector fields on (an open subset of) $\mathbb{R}^{2}$ and $\mathscr{G}$ its associated local Lie group of transformations (cf. $[18,19])$.

Given a transformation $g \in \mathscr{G}$, its derivative at a point $P \in \mathbb{R}^{2}$, denoted by $D g(P)$, is a linear map

$$
D g(P): T_{P} \mathbb{R}^{2} \rightarrow T_{g(P)} \mathbb{R}^{2}
$$


The projective transformation generated by $(2.1)$ will be denoted by

$$
g^{(1)}(P): \mathbb{P}\left(T_{P} \mathbb{R}^{2}\right) \rightarrow \mathbb{P}\left(T_{g(P)} \mathbb{R}^{2}\right),
$$

where $\mathbb{P}\left(T_{P} \mathbb{R}^{2}\right)$ denotes the projective space built on $T_{P} \mathbb{R}^{2}$; in other words,

$$
g^{(1)}(P) \cdot[\mathbf{v}]=[D g(P) \cdot \mathbf{v}], \quad \text { for all } \mathbf{v} \in T_{P} \mathbb{R}^{2}, \mathbf{v} \neq 0 .
$$

The mapping (2.2) is closely related to the notion of first prolongation $[3,17,18]$, of the transformation $g$. Indeed, if $g=\left(g_{1}, g_{2}\right), P=(x, y)$ and

$$
\mathbf{v}=(\dot{x}, \dot{y}) \in T_{P} \mathbb{R}^{2}
$$

is a non-vertical vector, then the affine coordinate of $[\mathbf{v}] \in \mathbb{P}\left(T_{p} \mathbb{R}^{2}\right)$ is given by

$$
y^{\prime}=\dot{y} / \dot{x},
$$

while that of $g^{(1)}(P) \cdot[\mathbf{v}]$ is given by

$$
\tilde{y}^{\prime}=\frac{g_{2, x}(x, y)+g_{2, y}(x, y) y^{\prime}}{g_{1, x}(x, y)+g_{1, y}(x, y) y^{\prime}}
$$

(cf. (2.1)-(2.3)). The first prolongation of $g$ at $\left(x, y, y^{\prime}\right)$ is then defined by

$$
\operatorname{pr}^{(1)} g\left(x, y, y^{\prime}\right)=\left(g(x, y), \tilde{y}^{\prime}\right) \text {. }
$$

Definition 2.1. A Lie algebra of vector fields $g$ is primitive in an open subset $U \subset \mathbb{R}^{2}$ if there is no one-dimensional foliation of $U$ left invariant by the action of $\mathscr{G}$; otherwise, $g$ is imprimitive in $U$. Equivalently, $g$ is primitive in $U$ if there is no one-dimensional distribution $\mathscr{D}$ on $U$ invariant under the action of the maps (2.1) or (2.2), for all $g \in \mathscr{G}$. Finally, $g$ is locally primitive if it is primitive in every open subset $U \subset \mathbb{R}^{2}$.

A primitive Lie algebra of vector fields may fail to be locally primitive, as discussed, for instance, in [7]. However, since this paper is concerned with questions of a local nature, the latter fact will not play an important role here.

Definition 2.2. A point $P \in \mathbb{R}^{2}$ will be called generic for the Lie algebra of vector fields $g$ if the dimension of the linear subspace of $T_{Q} \mathbb{R}^{2}$,

$$
\mathrm{g}(Q) \equiv\{X(Q) \mid X \in \mathfrak{g}\}
$$

is constant for all $Q$ in some neighbourhood of $P$. Equivalently, $P$ is a generic point for $g$ if and only if all the orbits of $\mathscr{G}$ have constant dimension in a neighbourhood of $P$.

For real analytic Lie algebras-which are the only ones we shall deal with in this paper-it is clear that the set of generic points is dense in $\mathbb{R}^{2}$.

If all the orbits of $\mathscr{G}$ in an open subset $U$ are two-dimensional, $\mathscr{G}$ and its Lie algebra $g$ are said to be transitive in $U$; otherwise, $\mathscr{G}$ and $g$ are intransitive in $U$. A well-known necessary and sufficient condition for $g$ to be transitive in a neighbourhood of a point $P \in \mathbb{R}^{2}$ is that

$$
\mathrm{g}(P)=T_{P} \mathbb{R}^{2}
$$

Notice that if a Lie algebra of vector fields $g$ is intransitive, then $g$ is clearly imprimitive in a neighbourhood of every generic point. 
Let $\mathscr{G}_{P} \subset \mathscr{G}$ denote the isotropy subgroup of $\mathscr{G}$ at $P \in \mathbb{R}^{2}$, that is,

$$
\mathscr{G}_{P}=\{g \in \mathscr{G} \mid g(P)=P\} .
$$

Its Lie algebra is the isotropy subalgebra of $g$ at $P$, given by

$$
\mathrm{g}_{P}=\{X \in \mathrm{g} \mid X(P)=0\} .
$$

Since $P$ is fixed by any transformation $g \in \mathscr{G}_{P}$, it follows that $D g(P)$ is a linear transformation of $T_{P} \mathbb{R}^{2}$. This defines an action

$$
\sigma: \mathscr{G}_{P} \times T_{P} \mathbb{R}^{2} \rightarrow T_{P} \mathbb{R}^{2}
$$

of $\mathscr{G}_{P}$ on $T_{P} \mathbb{R}^{2}$ by linear mappings, that is, a representation of $\mathscr{G}_{P}$ as a subgroup of $\operatorname{GL}\left(T_{P} \mathbb{R}^{2}\right)$. The representation (2.9) induces a representation

$$
\rho: g_{P} \times T_{P} \mathbb{R}^{2} \rightarrow T_{P} \mathbb{R}^{2}
$$

of $\mathfrak{g}$ as a subalgebra of $\mathrm{gl}\left(T_{P} \mathbb{R}^{2}\right)$ in the usual way:

$$
\rho(X, \mathrm{v}) \equiv X \cdot \mathrm{v}=\left.\frac{d}{d t}\right|_{t=0} \sigma(g(t), \mathrm{v}),
$$

where $\{g(t)\}_{t \in \mathbb{R}} \subset \mathscr{G}_{P}$ is the one-parameter subgroup generated by $X$. Identifying the tangent space to $T_{P} \mathbb{R}^{2}$ at $\mathbf{v}$ with $T_{P} \mathbb{R}^{2}$ itself and the vector field

$$
X=\xi(x, y) \partial_{x}+\eta(x, y) \partial_{y} \in \mathrm{g}_{P}
$$

with the maip $X: \mathbb{R}^{2} \rightarrow \mathbb{R}^{2}$ given by

$$
(x, y) \mapsto(\xi(x, y), \eta(x, y)),
$$

we obtain the following explicit formula for the representation (2.10):

$$
X \cdot \mathbf{v}=D X(P) \cdot \mathbf{v} \text {. }
$$

In other words, $X \in \mathrm{g}_{P}$ is represented by the linear map

$$
\rho(X) \equiv D X(P) \in \mathfrak{g l}\left(T_{P} \mathbb{R}^{2}\right) .
$$

Note that since $X \mapsto \rho(X)$ is a representation, we have

$$
[\rho(X), \rho(Y)]=\rho([X, Y]),
$$

a result that could be checked by a direct calculation using (2.14). Finally, using as coordinates of $\mathbf{v} \in T_{P} \mathbb{R}^{2}$ its components $(\dot{x}, \dot{y})$ in the natural basis, we can write (2.13) as follows:

$$
X \cdot \mathbf{v}=\left(D_{t} \xi\right) \frac{\partial}{\partial \dot{x}}+\left(D_{t} \eta\right) \frac{\partial}{\partial \dot{y}}
$$

where $D_{t}$ stands for the 'total time derivative' operator:

$$
D_{t}=\dot{x} \frac{\partial}{\partial x}+\dot{y} \frac{\partial}{\partial y} \text {. }
$$

In a completely analogous way, we have an action $\tilde{\sigma}$ of $\mathscr{G}_{P}$ on $\mathbb{P}\left(T_{P} \mathbb{R}^{2}\right)$ by projective transformations, given by

$$
g \in \mathscr{G}_{P} \mapsto g^{(1)}(P) .
$$

As before, this action induces an action $\tilde{\rho}$ of $\mathfrak{g}_{P}$ on $\mathbb{P}\left(T_{P} \mathbb{R}^{2}\right)$ by the formula

$$
\tilde{\rho}(X,[\mathbf{v}]) \equiv \tilde{\rho}(X) \cdot[\mathbf{v}] \equiv X \cdot[\mathbf{v}]=\left.\frac{d}{d t}\right|_{t=0}\left(g(t)^{(1)}(P) \cdot[\mathbf{v}]\right) .
$$


Taking into account (2.5a), we obtain the following explicit expression for the action (2.19):

with (cf. (2.4) and (2.16))

$$
X \cdot[\mathbf{v}]=\left(a y^{\prime 2}+b y^{\prime}+c\right) \frac{\partial}{\partial y^{\prime}},
$$

$$
a=-\xi_{y}(P), \quad b=\eta_{y}(P)-\xi_{x}(P), \quad c=\eta_{x}(P) .
$$

As was to be expected from the analogous fact for $g^{(1)}(P), \tilde{\rho}(X)$ is closely related to the first prolongation $\mathrm{pr}^{(1)} X$ of the vector field $\mathbf{X}$ (cf. [18]); in fact, we have

$$
\operatorname{pr}^{(1)} X\left(x, y, y^{\prime}\right)=X(x, y) \oplus \tilde{\rho}(X,[\mathbf{v}]) .
$$

In other words, $\tilde{\rho}(X,[\mathbf{v}])$ is the $y^{\prime}$ component of $\mathrm{pr}^{(1)} X$ at $\left(x, y, y^{\prime}\right)$. An important property of $\tilde{\rho}$ worth pointing out is that $\tilde{\rho}\left(\mathrm{g}_{p}\right)$ is a Lie algebra of vector fields on $\mathbb{P}\left(T_{P} \mathbb{R}^{2}\right)$ and $\tilde{\rho}$ is a Lie algebra homomorphism, that is,

$$
[\tilde{\rho}(X), \tilde{\rho}(Y)]=\tilde{\rho}([X, Y]) \text {. }
$$

As before, the latter formula can be checked directly by a straightforward calculation; it is, of course, equivalent to the following familiar property of the first prolongation of vector fields:

$$
\left[\mathrm{pr}^{(1)} X, \mathrm{pr}^{(1)} Y\right]=\mathrm{pr}^{(1)}[X, Y] \text {. }
$$

The distinction between primitive and imprimitive algebras plays a key role in Lie's local classification of Lie algebras of vector fields in $\mathbb{C}^{2}$ [15]. (See also $[2,5,9]$.) Roughly speaking, the fact that a Lie algebra of vector fields is imprimitive means that the action on $\mathbb{C}^{2}$ or $\mathbb{R}^{2}$ of its local group of transformations can be understood in terms of its action on a cross-section of its invariant one-dimensional foliation. Since this cross-section has (complex or real) dimension 1, this explains intuitively why the local classification of the imprimitive algebras of vector fields in $\mathbb{C}^{2}$ or $\mathbb{R}^{2}$ can be reduced to the local classification of Lie algebras of vector fields in $\mathbb{C}$ or $\mathbb{R}$. Now, it is well known [15], that in one dimension there is no difference between the real and the complex classifications: in either case, the only possible examples of Lie algebras of vector fields are, up to a local change of variables, the subalgebras of the three-dimensional (real or complex) projective group. These heuristic considerations strongly suggest that the classification of imprimitive Lie algebras of vector fields in the plane is the same in the real and the complex cases. Indeed, this can be rigorously verified by going through the lengthy, though elementary, calculation found in [15] for the imprimitive case and checking that it still holds when the coordinates $(x, y)$ in which the calculation is carried out are assumed to be real instead of complex. (See also [9].) We thus have the following theorem:

THEOREM 2.3. If $\mathrm{g}$ is an imprimitive Lie algebra of vector fields in an open subset of $\mathbb{R}^{2}$, then there are local coordinates $(x, y)$ in $\mathbb{R}^{2}$ in which $\mathfrak{g}$ assumes one of the forms listed in Table 1.II.

To finish the local classification of Lie algebras of vector fields in $\mathbb{R}^{2}$, we can thus restrict ourselves from now on to the class of locally primitive algebras. Before we start classifying these algebras, however, it is convenient to derive several criteria for checking primitivity that will be useful in the following discussion. 
Definition 2.4. We shall say that the action of $\mathscr{G}_{P}$ (respectively $g_{P}$ ) on $T_{P} \mathbb{R}^{2}$ is reducible if there is a proper (i.e. one-dimensional) linear subspace of $T_{P} \mathbb{R}^{2}$ invariant under $\mathscr{G}_{P}$ (respectively $\mathrm{g}_{P}$ ).

Proposition 2.5. Let $P$ be a generic point for a Lie algebra, $\mathrm{g}$, of vector fields in $\mathbb{B}^{2}$. The following facts are then equivalent:

(1) $\mathrm{g}$ is imprimitive in a neighbourhood of $P$;

(2) the action of $\mathscr{G}_{P}$ on $T_{P} \mathbb{R}^{2}$ is reducible;

(3) the action of $g_{P}$ on $T_{P} \mathbb{R}^{2}$ is reducible;

(4) the action of $\mathscr{G}_{P}$ on $\mathbb{P}\left(T_{P} \mathbb{R}^{2}\right)$ has at least one fixed point;

(5) $\tilde{\rho}\left(\mathrm{g}_{P}\right)$ vanishes at least at one point of $\mathbb{P}\left(T_{P} \mathbb{R}^{2}\right)$.

Proof. First of all, it is clear that statements (3) and (5) are the infinitesimal versions of (2) and (4), respectively, so that it suffices to prove the equivalence of (1), (2) and (4). Secondly, since the equivalence of (2) and (4) is obvious, we shall only show here that (1) is equivalent to (2).

$(1) \Rightarrow(2)$. If $g$ is imprimitive in some neighbourhood $U$ of $P$, then there is by definition a one-dimensional distribution $\mathscr{D}$ invariant under the action of $\mathscr{G}$. Let

$$
L=\mathscr{D}(P) \subset T_{P} \mathbb{R}^{2} .
$$

Then $L$ is clearly invariant under the action of $\mathscr{G}_{P}$, since by the invariance of $\mathscr{D}$ under the action of $\mathscr{G}$ we have

$$
\sigma(g) \cdot L \equiv \sigma(g) \cdot \mathscr{D}(P)=\mathscr{D}(g(P)), \quad \text { for all } g \in \mathscr{G},
$$

and

$$
\mathscr{D}(g(P))=\mathscr{D}(P) \equiv L, \quad \text { for all } g \in \mathscr{G}_{P},
$$

by definition of $\mathscr{G}_{P}$.

$(2) \Rightarrow(1)$. Suppose now that there are a generic point $P \in \mathbb{R}^{2}$ and a proper linear subspace $L \subset T_{P} \mathbb{R}^{2}$ invariant under the action of $\mathscr{G}_{P}$, that is,

$$
\sigma(g) \cdot \mathbf{v}=\lambda(g, \mathbf{v}) \mathbf{v}, \quad \text { for all } g \in \mathscr{G}_{P}, \mathbf{v} \in L .
$$

If $\mathscr{G}$ is intransitive on a neighbourhood of $P$, we have finished, by the remarks following Definition 2.2. Otherwise, let $U$ be an open neighbourhood of $P$ on which $\mathscr{G}$ is transitive. If $Q \in U$, there is at least one transformation $g_{Q} \in \mathscr{G}$ such that

$$
g_{Q}(P)=Q \text {. }
$$

We can then define a one-dimensional distribution $\mathscr{D}$ on $U$ by setting

$$
\mathscr{D}(Q)=D g_{Q}(P) \cdot L \text {. }
$$

To verify that $\mathscr{D}$ is well defined, we have to check that the right-hand side of the latter formula does not depend on the choice of the transformation $g_{Q}$ satisfying (2.28). But this is easy, since if $h \in \mathscr{G}$ satisfies $h(P)=Q$ then

$$
h=g_{Q} g_{P}
$$

with $g_{P} \in \mathscr{G}_{P}$. We then have

$$
\begin{aligned}
D h(P) \cdot L & =D g_{Q}(P) D g_{P}(P) \cdot L \\
& \equiv D g_{Q}(P)\left(\sigma\left(g_{P}\right) \cdot L\right)=D g_{Q}(P) \cdot L,
\end{aligned}
$$


where the last equality is a consequence of the invariance of $L$ under $\mathscr{G}_{P}$. Finally, the fact that the distribution $\mathscr{D}$ we have just defined is invariant under the action of $\mathscr{G}_{P}$ is also straightforward, since we have

$$
\begin{aligned}
D g(Q) \cdot \mathscr{D}(Q) & \equiv D g(Q) D g_{Q}(P) \cdot L=D\left(g \circ g_{Q}\right)(P) \cdot L \\
& \equiv \mathscr{D}\left(\left(g \circ g_{Q}\right)(P)\right)=\mathscr{D}(g(Q)), \quad \text { for all } g \in \mathscr{G}
\end{aligned}
$$

This completes the proof of the proposition.

CoRollary 2.6. If there is a generic point $P \in \mathbb{R}^{2}$ such that $\tilde{\sigma}\left(\mathscr{G}_{P}\right)$ is the full projective group of $\mathbb{P}\left(T_{P} \mathbb{R}^{2}\right)$, then $g$ is primitive in an open neighbourhood of $P$.

Proof. Indeed, since the projective group has obviously no fixed points, the result follows from the equivalence between statements (1) and (4) of Proposition 2.5 .

At this point, we would like to remark that Proposition 2.5 and Corollary 2.6 are also valid in the complex case. On the other hand, whereas the converse of Corollary 2.6 is true in the complex case [2, § 147], it is generally false in the real one. This makes a crucial difference, since the whole classical classification of the primitive algebras in $\mathbb{C}^{2}$ depends critically on the validity of the converse of the latter corollary. Although the reason of why Corollary 2.6 does not hold in the real case will become apparent in the next section, it is appropriate to illustrate it here by means of a very simple example.

EXAMPLE 2.7. Let $g$ be the Lie algebra of the group $\mathscr{G}$ of dilations and motions of $\mathbb{R}^{2}$, generated by the vector fields

$$
\partial_{x}, \partial_{y}, x \partial_{x}+y \partial_{y}, y \partial_{x}-x \partial_{y}
$$

In this example, it is clear that every point is generic, the dimension of $g(P)$ being equal to 2 everywhere. Also, it is plain that $g$ is locally primitive, since for every $P \in \mathbb{R}^{2}$ the subgroup of rotations around $P$ acts irreducibly on $T_{P} \mathbb{R}^{2}$. However, $\tilde{\sigma}\left(\mathscr{G}_{P}\right)$ is never the full projective group, since it is only one-dimensional for every $P \in \mathbb{R}^{2}$. Indeed, the isotropy subgroup of a point $P$ is the two-dimensional subgroup generated by the rotations and the dilations around that point; however, when we consider the action of this subgroup on the projective space $\mathbb{P}\left(T_{P} \mathbb{R}^{2}\right)$, it is obvious that the dilations around $P$ act as the identity transformation.

On the other hand, if we regard (2.33) as a Lie algebra of vector fields in the complex plane, the situation is totally different. Indeed, in this case (2.33) is no longer primitive, since it can be easily checked that the family of parallel lines

$$
y=i x+c, \quad \text { with } c \in \mathbb{C},
$$

gives a one-dimensional foliation invariant under dilations and motions of $\mathbb{C}^{2}$.

\section{Locally primitive algebras: generalities}

Lie proved in [15] that there are exactly the following three local equivalence classes of (locally) primitive Lie algebras of vector fields in $\mathbb{C}^{2}$, up to changes of analytic coordinates:

$$
\begin{aligned}
& \operatorname{span}\left\{p, q, x p, y p, x q, y q, x^{2} p+x y q, x y p+y^{2} q\right\} \\
& \operatorname{span}\{p, q, x p, y p, x q, y q\} \\
& \operatorname{span}\{p, q, x p-y q, x q, y p\}
\end{aligned}
$$


where we are using the convenient classical notation

$$
p \equiv \partial_{x}, \quad q \equiv \partial_{y},
$$

and where $\operatorname{span}\left\{X_{1}, \ldots, X_{n}\right\}$ denotes the Lie algebra with basis $\left\{X_{1}, \ldots, X_{n}\right\}$. The corresponding Lie groups are the full projective group, the general affine group, and the special affine group, respectively.

Of course, if $(x, y)$ are real instead of complex coordinates, the algebras (3.1) are examples of locally primitive Lie algebras of vector fields in the real plane. On the other hand, from Example 2.7 we know that the Lie algebras (3.1) do not exhaust all the possible equivalence classes of locally primitive Lie algebras in $\mathbb{R}^{2}$. In this section we shall start the classification of locally primitive Lie algebras of vector fields in the real plane by studying their linearization at a generic point, as a first step for finding all the local equivalence classes of these algebras not already included in the list (3.1).

Proposition 3.1. A Lie algebra $g$ of vector fields in $\mathbb{R}^{2}$ is locally equivalent under a real analytic change of coordinates to one of the algebras (3.1) if and only if there is a generic point $P$ such that

$$
\operatorname{dim} \tilde{\rho}\left(\mathfrak{g}_{P}\right)=3 .
$$

Proof. First of all, it can be readily checked that for any of the Lie algebras (3.1), $\bar{\rho}\left(g_{P}\right)$ is three-dimensional for all $P \in \mathbb{R}^{2}$, in agreement with the remarks following Corollary 2.6. Conversely, if $\tilde{\rho}\left(\mathrm{g}_{P}\right)$ is three-dimensional then $\mathrm{g}$ is primitive in a neighbourhood of $P$ by Corollary 2.6. Moreover, it can be shown that when (3.3) is satisfied, Lie's calculations for the complex primitive case (cf. $[15$, pp. $72-87 ; 2$, pp. 388-400]) carry over without modification to the real primitive case.

Proposition 3.2. If $\mathrm{g}$ is a locally primitive Lie algebra of vector fields in $\mathbb{R}^{2}$ not locally equivalent under a real analytic change of coordinates to one of the algebras (3.1), then

$$
\operatorname{dim} \tilde{\rho}\left(g_{P}\right)=1
$$

at every generic point $P$.

Proof. By the previous proposition, all we have to do is to check that $\tilde{\rho}\left(\mathrm{g}_{P}\right)$ cannot be zero- or two-dimensional at a generic point $P$. The proof of this simply consists of an elementary case-by-case analysis.

First of all, it is clear that $\tilde{\rho}\left(\mathrm{g}_{P}\right)$ cannot be zero at a generic point $P$, since otherwise statement (5) of Proposition 2.5 would be trivially satisfied, and therefore $g$ would be imprimitive in a neighbourhood of such a point $P$.

Assume, therefore, that $\tilde{\rho}\left(g_{P}\right)$ is two-dimensional at a generic point $P$, and let

$$
U=\left(a y^{\prime 2}+b y^{\prime}+c\right) \frac{\partial}{\partial y^{\prime}}, \quad V=\left(\alpha y^{\prime 2}+\beta y^{\prime}+\gamma\right) \frac{\partial}{\partial y^{\prime}}
$$

be a basis of $\tilde{\rho}\left(g_{P}\right)$. We shall then show that the vector fields (3.5) must necessarily have a common zero, which by the equivalence of statements (1) and (5) of Proposition 2.5 would again imply that $g$ is imprimitive in a neighbourhood of $P$. 
To begin with, it is clear that we can assume without loss of generality that $c=0$, replacing, if necessary, $U$ by a suitable linear combination of $U$ and $V$. Suppose first that

(i) $a=0$.

We can then let $b=1, \beta=0$ without loss of generality, and hence

$$
[U, V]=\left(\alpha y^{\prime 2}-\gamma\right) \frac{\partial}{\partial y^{\prime}}
$$

The right-hand side of (3.7) is a linear combination of $U$ and $V$ if and only if $\alpha \gamma=0$, that is, if either

or

$$
V=y^{\prime 2} \frac{\partial}{\partial y^{\prime}}
$$

$$
V=\frac{\partial}{\partial y^{\prime}} .
$$

In the first instance, both $U$ and $V$ vanish at the horizontal line element $\left(y^{\prime}=0\right)$. In the second one, changing coordinates in $\mathbb{P}\left(T_{P} \mathbb{R}^{2}\right)$ by setting

$$
x^{\prime}=1 / y^{\prime},
$$

we easily check that

$$
U=-x^{\prime} \frac{\partial}{\partial x^{\prime}}, \quad V=-x^{\prime 2} \frac{\partial}{\partial x^{\prime}},
$$

which vanish simultaneously at the vertical line element $x^{\prime}=0$.

Finally, suppose that

(ii) $a \neq 0$.

In this case, we can take $a=1, \alpha=0$. If $\gamma=0, U$ and $V$ both vanish at $y^{\prime}=0$. On the other hand, if $\gamma \neq 0$, we can further normalize $V$ by setting $\gamma=1$, and we have

$$
[U, V]=-\left(\beta y^{\prime 2}+2 y^{\prime}+b\right) \frac{\partial}{\partial y^{\prime}} .
$$

The right-hand side of the latter equation is a linear combination of $U$ and $V$ if and only if

$$
b \beta=1 \text {, }
$$

that is, if and only if (up to immaterial constant factors)

$$
U=\left(y^{\prime 2}+b y^{\prime}\right) \frac{\partial}{\partial y^{\prime}}, \quad V=\left(y^{\prime}+b\right) \frac{\partial}{\partial y^{\prime}},
$$

which vanish simultaneously at $y^{\prime}=-b$. This completes the proof of the proposition.

It should now be clear why the converse of Corollary 2.6 is true in the complex case. Indeed, if $\tilde{\rho}\left(\mathrm{g}_{P}\right)$ is generated by only one vector field, then it necessarily has a zero in the complex case, by (2.20) and the Fundamental Theorem of Algebra. 
The equivalence between statements (1) and (5) of Proposition 2.5 would then imply that $g$ is imprimitive in a neighbourhood of $P$.

Our next step shall be to take advantage of the previous proposition to determine the forms, up to terms of order 2 or higher, of all locally primitive Lie algebras of vector fields in $\mathbb{R}^{2}$ not equivalent to one of the algebras (3.1). To this end, we introduce the notion of the order of a vector field at a point, which will play an important role in all that follows:

Definition 3.3. If $X$ is a vector field real analytic at $P \equiv(a, b) \in \mathbb{R}^{2}$, we shall say that $X$ is of order greater than or equal to $k$ at $P$ if

that is,

$$
X=o\left((|x-a|+|y-b|)^{k-1}\right),
$$

$$
\lim _{(x, y) \rightarrow(a, b)} \frac{X(x, y)}{(|x-a|+|y-b|)^{k-1}}=0 .
$$

In the latter formula, we assume for convenience that $(x, y)$ are the standard Cartesian coordinates of $\mathbb{R}^{2}$ and $X(x, y)$ is the mapping $\mathbb{R}^{2} \rightarrow \mathbb{R}^{2}$ naturally associated to $X$, as explained in the previous section. However, it is not difficult to show that the above definition is actually independent of the (real analytic) coordinates used around $P$. In particular, it is no loss of generality to assume that the coordinates of $P$ are $(0,0)$, as we shall do from now on.

If $P$ is a generic point for a real analytic Lie algebra of vector fields $g$ in $\mathbb{R}^{2}$, let us define the sets

$$
\mathrm{g}_{P}^{k}=\{X \in \mathfrak{g} \mid X \text { is of order at least } k \text { at } P\}
$$

for $k \geqslant 0$. In particular,

$$
\mathrm{g}_{P}^{0}=\mathrm{g}, \quad \mathrm{g}_{P}^{1}=\mathrm{g}_{P}
$$

and we also have

$$
\mathrm{g}_{P}^{j} \subset \mathrm{g}_{P}^{k}, \quad \text { for all } j \geqslant k .
$$

Moreover, from the equations

$$
\left[\mathrm{g}_{P}^{j}, \mathrm{~g}_{P}^{k}\right] \subset \mathrm{g}_{P}^{j+k-1} \quad\left(j^{2}+k^{2}>0\right),
$$

it follows that $\mathrm{g}_{P}^{j}$ is a subalgebra of $\mathrm{g}$ for all $j$, and that $\mathrm{g}_{P}^{j}$ is an ideal of $\mathrm{g}_{P}^{k}$ for all $1 \leqslant k \leqslant j$.

Thus, (3.14) and (3.17) define a filtration

$$
\mathfrak{g}_{P}^{0} \equiv \mathfrak{g} \supset \mathfrak{g}_{P}^{1} \equiv \mathfrak{g}_{P} \supset \ldots \supset \mathrm{g}_{P}^{j} \supset \mathrm{g}_{P}^{j+1} \supset \ldots
$$

of $g$. Since $g_{P}^{2}$ is an ideal of $g_{P}$, the quotient $g_{P} / g_{P}^{2}$ is a Lie algebra, with Lie bracket

$$
\left[X+\mathrm{g}_{P}^{2}, Y+\mathrm{g}_{P}^{2}\right]=[X, Y]+\mathrm{g}_{P}^{2}, \quad \text { for all } X, Y \in \mathrm{g}_{P} .
$$

We shall call

$$
\mathscr{L}_{P}(\mathrm{~g}) \equiv \mathrm{g}_{P} / \mathrm{g}_{P}^{2}
$$

the linear part of $g$ at $P$, since it can be identified to the space obtained by taking the vector fields in $g$ whose order at $P$ is 1 and discarding the terms of order 2 or 
higher. To make this a little more precise, let $\mathscr{L}$ be the Lie algebra of linear vector fields in $\mathbb{R}^{2}$, and define a mapping

by

$$
\varphi: \mathscr{L}_{P}(\mathrm{~g}) \rightarrow \mathscr{L}
$$

$$
\varphi(X)=\text { linear part of } X \text { at } P \text {. }
$$

For instance, if $P=(0,0)$ and $X=\left(x-y^{2}\right) p+\sin (2 y) q$ then

$$
\varphi(X)=x p+2 y q .
$$

It is then easy to show that $\mathscr{L}_{P}(\mathrm{~g})$ is isomorphic to the subalgebra of linear vector fields $\varphi\left(\mathscr{L}_{P}(\mathrm{~g})\right)$.

We shall now compute $\mathscr{L}_{P}(\mathrm{~g})$ for all locally primitive Lie algebras not equivalent to one of the algebras (3.1) under a real analytic change of local coordinates. To this end, notice that by $(2.21)$ we have

$$
\bar{\rho}\left(g_{P}^{2}\right)=0 .
$$

The map $\tilde{\rho}$ thus passes to the quotient, i.e. we can define a map (also called $\tilde{\rho}$ )

$$
\tilde{\rho}: \mathscr{L}_{P}(\mathfrak{g}) \rightarrow \tilde{\rho}\left(\mathfrak{g}_{P}\right)
$$

by setting

$$
\tilde{\rho}\left(X+g_{P}^{2}\right)=\tilde{\rho}(X) .
$$

By elementary linear algebra,

$$
\operatorname{dim} \mathscr{L}_{P}(\mathrm{~g})=\operatorname{dim} \operatorname{ker} \tilde{\rho}+\operatorname{dim} \tilde{\rho}\left(\mathrm{g}_{P}\right)=\operatorname{dim} \operatorname{ker} \tilde{\rho}+1,
$$

where the last equality is a consequence of Proposition 3.2. Finally, suppose that

$$
X+\mathrm{g}_{P}^{2} \in \operatorname{ker} \tilde{\rho} .
$$

By (2.21), this implies that

$$
\xi_{y}(P)=\eta_{x}(P)=0, \quad \xi_{x}(P)=\eta_{y}(P)
$$

and therefore $X$ is of the form

$$
X=\lambda(x p+y q)+\ldots,
$$

where of course $\lambda=\xi_{x}(P)$ and the dots stand for terms of order 2 or higher at $P$. We thus see that $\operatorname{ker} \bar{\rho}$ is either zero- or one-dimensional. We have thus proved the following:

Proposition 3.4. If $\mathrm{g}$ is a locally primitive Lie algebra of vector fields in $\mathbb{R}^{2}$ not equivalent to an algebra of type (3.1), then its linear part $\mathscr{L}_{P}(\mathrm{~g})$ at a generic point $P$ is either one- or two-dimensional. Moreover, $\mathscr{L}_{P}(\mathrm{~g})$ is two-dimensional if and only if $\mathrm{g}$ contains $a$ vector field agreeing with $x p+y q$ up to terms of order 2 or higher at $P$.

To conclude this section, we are going to find normal forms for $\mathscr{L}_{P}(\mathrm{~g})$ when, as before, $g$ is a locally primitive algebra of vector fields not locally equivalent to any of the algebras (3.1). According to the previous proposition, there are two cases to consider:

(i) $\operatorname{dim} \mathscr{L}_{P}(\mathrm{~g})=1$. 
In this case, let $X$ be the generator of $\mathscr{L}_{P}(\mathrm{~g})$, and consider the linear part of $X$,

$$
X_{L} \equiv \varphi(X)=(a x+b y) p+(c x+d y) q
$$

(cf. (3.21)). By (2.21),

$$
\bar{\rho}(X)=\left[c+(d-a) y^{\prime}-b y^{\prime 2}\right] \frac{\partial}{\partial y^{\prime}},
$$

and since $\tilde{\rho}\left(g_{P}\right)$ is one-dimensional by Proposition 3.2 , it must be generated by (3.29). But, by the equivalence between (1) and (5) in Proposition 2.5, the latter vector field must have no zeros in $\mathbb{P}\left(T_{P} \mathbb{R}^{2}\right)$, which is only possible if

$$
(d-a)^{2}+4 b c<0
$$

Calling

$$
A=\left(\begin{array}{ll}
a & b \\
c & d
\end{array}\right)
$$

the matrix of the coefficients of the linear vector field (3.28), the latter equality is equivalent to

$$
(\operatorname{tr} A)^{2}<4 \operatorname{det} A .
$$

Thus the real canonical form of $A$ is

$$
M^{-1} A M=\lambda\left(\begin{array}{cc}
\alpha & 1 \\
-1 & \alpha
\end{array}\right)
$$

It is then immediate to check that if we perform the linear change of coordinates

$$
(\bar{x}, \bar{y})=M \cdot(x, y)
$$

then we obtain

$$
X=\alpha\left(\bar{x} \frac{\partial}{\partial \bar{x}}+\bar{y} \frac{\partial}{\partial \bar{y}}\right)+\left(\bar{y} \frac{\partial}{\partial \bar{x}}-\bar{x} \frac{\partial}{\partial \bar{y}}\right)+\ldots,
$$

where the dots represent terms of order higher than the first in $(\bar{x}, \bar{y})$ and we have suppressed the irrelevant factor $\lambda$. Since the order of a vector field at a point $P$ does not depend on the real analytic coordinates chosen around $P$, the new coordinates $(\bar{x}, \bar{y})$ are as good as the old ones. We have thus shown that when $\mathscr{L}_{P}(\mathrm{~g})$ is one-dimensional there is a coordinate system around $P$ in which the generator of $\mathscr{L}_{P}(\mathrm{~g})$ assumes the simple form

$$
X=\alpha(x p+y q)+(y p-x q)+\ldots .
$$

(ii) $\operatorname{dim} \mathscr{L}_{P}(\mathrm{~g})=2$.

First of all, we know from Proposition 3.4 that one of the generators of $\mathscr{L}_{P}(\mathrm{~g})$ is of the form

$$
Y=x p+y q+\ldots,
$$

where the dots stand for terms of order higher than 1 at $P \equiv(0,0)$. Let $X$ be the second generator, with $\varphi(X)$ given again by (3.28). Since

$$
\tilde{\rho}(Y)=0,
$$


we see that $\tilde{\rho}\left(g_{P}\right)$ is again generated by $\tilde{\rho}(X)$. Hence, exactly as in the previous case, there is a linear change of coordinates (3.34) under which $X$ assumes the form (3.35). On the other hand, it is plain that any linear change of coordinates will turn (3.37) into

$$
Y=\bar{x} \frac{\partial}{\partial \bar{x}}+\bar{y} \frac{\partial}{\partial \bar{y}}+\ldots
$$

where as usual the dots stand for terms of order higher than 1 in $(\bar{x}, \bar{y})$. Choosing $Y$ and $X-\alpha Y$ as the new basis of $\mathscr{L}_{P}(\mathrm{~g})$ and dropping the overbars, we see that in this case $\mathscr{L}_{P}(\mathfrak{g})$ is generated by the vector fields

$$
x p+y q+\ldots, \quad y p-x q+\ldots .
$$

Remark 3.5. The results we have found so far allow us to give a complete description of $\mathrm{g}$ up to terms of order 2 or higher at a generic point $P$. Before doing this, it is perhaps necessary to point out that the set

$$
\mathrm{g}-\mathrm{g}_{P}^{k+1}
$$

of all vector fields in $\mathrm{g}$ which are of order $k$ or less at a generic point $P$ is obviously not a linear space. However, it is natural to regard two elements of the previous set as equivalent when they differ by a vector field in $g$ whose order at $P$ is greater than or equal to $k+1$. The quotient space obtained from (3.41) in this way is the linear space $\mathrm{g} / \mathrm{g}_{P}^{k+1}$. It is customary in the classical literature to identify both spaces, and thus, for example, to speak of a basis of (3.41), when what is really meant is a set of vector fields in (3.41) whose equivalence classes form a basis of $\mathrm{g} / \mathrm{g}_{P}^{k+1}$. Since this common practice is almost always harmless and notationally quite convenient, we shall adopt it in what follows.

THEOREM 3.6. If $\mathrm{g}$ is a locally primitive Lie algebra of vector fields in $\mathbb{R}^{2}$ not locally equivalent under a real analytic change of coordinates to one of the algebras (3.1), we can choose local coordinates around a point $P$ generic for $g$ such that $\mathrm{g}-\mathrm{g}_{P}^{2}$ is generated either by

$$
p+\ldots, \quad q+\ldots, \quad \alpha(x p+y q)+(y p-x q)+\ldots
$$

or by

$$
p+\ldots, \quad q+\ldots, \quad x p+y q+\ldots, \quad y p-x q+\ldots .
$$

(In the latter formulas, the dots stand for terms of higher order at $x=y=0$ than those preceding them; as usual, we have assumed that $P$ has coordinates $(0,0)$.)

Proof. First of all, notice that by (2.6), g contains two vector fields linearly independent at $P$. By taking suitable linear combinations of these two vector fields, we see that $g$ contains the vector fields

$$
p+\ldots, q+\ldots,
$$

where the dots are terms of order 1 or higher at $P$. The latter vector fields are a basis for the vector fields of order zero at $P$, that is (cf. Remark 3.5 above), their equivalence classes modulo $g_{P}$ are a basis for $g / g_{P}$. Finally, since

$$
\mathrm{g} / \mathrm{g}_{P}^{2} \approx\left(\mathrm{g} / \mathrm{g}_{P}\right) \oplus\left(\mathrm{g}_{P} / \mathrm{g}_{P}^{2}\right) \equiv\left(\mathrm{g} / \mathrm{g}_{P}\right) \oplus \mathscr{L}_{P}(\mathrm{~g}),
$$

the theorem follows from the results about $\mathscr{L}_{P}(\mathrm{~g})$ obtained above. 


\section{Non-semisimple locally primitive algebras}

We shall determine in this section all the equivalence classes of locally primitive non-semisimple Lie algebras of vector fields in $\mathbb{R}^{2}$ not already included in Lie's list (3.1). The fundamental theorem needed to carry out this classification is the following:

THEOREM 4.1 [7]. If $\mathrm{g}$ is a non-semisimple locally primitive Lie algebra of vector fields in $\mathbb{R}^{2}$, and $P$ is a generic point for $\mathrm{g}$, then there exists a two-dimensional abelian ideal $a$ of $g$ such that:

(i) $\mathfrak{g}=\mathfrak{g}_{P} \oplus \mathfrak{a}$;

(ii) $g_{P}$ acts faithfully and irreducibly on $a$, that is,

(1) if $X \in \mathfrak{g}_{P}$ and $[X, \mathfrak{a}]=0$, then $X=0$,

(2) if $Y \in \mathfrak{a}$ and $[X, Y]=\lambda(X) Y$ for all $X \in \mathfrak{g}_{P}$, then $Y=0$.

We are now ready to classify all non-semisimple locally primitive Lie algebras of vector fields in $\mathbb{R}^{2}$ :

THEOREM 4.2. Let $\mathrm{g}$ be a non-semisimple locally primitive Lie algebra of vector fields in $\mathbb{R}^{2}$ not locally equivalent to one of the algebras (3.1). Then there are local coordinates $(x, y)$ in which either

$$
\mathrm{g}=\operatorname{span}\{p, q, \alpha(x p+y q)+y p-x q\}
$$

for some $\alpha \in \mathbb{R}$, or

$$
\mathfrak{g}=\operatorname{span}\{p, q, x p+y q, y p-x q\}
$$

Proof. To begin with, let $P$ be a generic point for $g$, and let us choose coordinates such that $P \equiv(0,0)$. By the previous theorem,

$$
\mathfrak{g}=\mathfrak{g}_{P} \oplus \mathfrak{a},
$$

where $\mathfrak{a}$ is an abelian ideal of $\mathrm{g}$. By the transitivity of $\mathrm{g}$ around $P$ we know (cf. (2.6)) that

$$
\mathrm{g} / \mathrm{g}_{P} \approx \mathbb{B}^{2}
$$

from which we deduce that

$$
\operatorname{dim} \mathfrak{a}=\operatorname{dim} \mathrm{g} / \mathrm{g}_{P}=2 \text {. }
$$

Moreover, from (4.3) and (2.6) it also follows that

$$
\operatorname{dim} a(P)=\operatorname{dim} g(P)=2 .
$$

From (4.5), (4.6) and the fact that $a$ is abelian, we can choose local coordinates around $P$ such that $P \equiv(0,0)$ and

$$
\mathfrak{a}=\operatorname{span}\{p, q\} .
$$

We now claim that

$$
\mathrm{g}_{P}^{2}=\{0\}
$$

Indeed, since $a$ is an ideal of $g$, we must have $\left[g_{P}^{2}, a\right] \subset a$, and from (3.17), $\left[\mathfrak{g}_{P}^{2}, \mathfrak{a}\right] \subset \mathfrak{g}_{P}$. Hence

$$
\left[g_{P}^{2}, a\right] \subset g_{P} \cap a=\{0\},
$$


from which (4.8) follows using Part (ii)(1) of the previous theorem. From this fact and Theorem 3.6, we can conclude that $g$ is spanned in a neighbourhood of $P$ either by

$$
p, q, \alpha(x p+y q)+y p-x q+U
$$

for some $\alpha \in \mathbb{R}$, or by

$$
p, q, x p+y q+D, y p-x q+R,
$$

where $U, D$ and $R$ are vector fields of order 2 or greater at $(0,0)$. (Notice that the change of variables used in Theorem 3.6 to bring $g_{P}$ to its canonical form (3.42) or (3.43) is linear, and therefore does not change (4.7).) Imposing now the condition that $\operatorname{span}\{p, q\}$ is an ideal of $\mathrm{g}$, we easily obtain that

$$
U=D=R=0 \text {. }
$$

Finally, we have already checked in $\S 2$ (cf. Example 2.7) that the Lie algebra (4.2) is indeed locally primitive. For the algebras of the form (4.1), it suffices to notice that the action of $g_{P}$ on $T_{P} \mathbb{R}^{2}$ is obviously given by multiplication by the matrix

$$
\left(\begin{array}{cc}
\alpha & 1 \\
-1 & \alpha
\end{array}\right)
$$

for all $P \in \mathbb{R}^{2}$. Since this matrix has no real eigenvalues, Proposition 2.5 implies that (4.1) is locally primitive.

Since the algebra (4.1) with parameter $-\alpha$ is related to the one with parameter $\alpha$ by the change of coordinates

$$
(x, y) \mapsto(-x, y),
$$

we can restrict $\alpha$ in (4.1) to assume only non-negative values. If we do this, then no two Lie algebras of the type (4.1) are equivalent under a local change of variables. Indeed, suppose that (4.1) is transformed into the Lie algebra

$$
\operatorname{span}\left\{\partial_{u}, \partial_{v}, \beta\left(u \partial_{u}+v \partial_{v}\right)+v \partial_{u}-u \partial_{v}\right\}
$$

by a local change of variables

$$
(u, v)=\Phi(x, y)
$$

Letting

$$
\Phi(0,0)=(a, b)
$$

be the $(u, v)$-coordinates of the point $P$, we can immediately check that the isotropy algebra of this point is generated by the vector field

$$
\beta\left(\xi \partial_{u}+\eta \partial_{v}\right)+\eta \partial_{u}-\xi \partial_{v},
$$

where $\xi=u-a, \eta=v-b$. Since both (4.18) and $\alpha(x p+y q)+y p-x q$ generate the isotropy subalgebra $g_{P}$, we must have

$$
\beta\left(\xi \partial_{u}+\eta \partial_{v}\right)+\eta \partial_{u}-\xi \partial_{v}=\lambda[\alpha(x p+y q)+y p-x q]
$$

for some $\lambda \neq 0$. Now, the action of (4.18) on $T_{P} \mathbb{B}^{2}$ is given by the matrix

$$
\left(\begin{array}{cc}
\beta & 1 \\
-1 & \beta
\end{array}\right)
$$


from (4.13), (4.16) and (4.19) we then easily obtain

with

$$
\left(\begin{array}{cc}
\beta & 1 \\
-1 & \beta
\end{array}\right)=\lambda M\left(\begin{array}{cc}
\alpha & 1 \\
-1 & \alpha
\end{array}\right) M^{-1}
$$

$$
M=D \Phi(0,0) .
$$

In particular, equating the trace and determinant of both sides of (4.21) gives the following system of equations:

$$
\begin{aligned}
\beta & =\lambda \alpha, \\
1+\beta^{2} & =\lambda^{2}\left(1+\alpha^{2}\right) .
\end{aligned}
$$

Eliminating the unknown quantity $\lambda$ among these equations yields

$$
\alpha^{2}=\beta^{2}
$$

which establishes our claim.

\section{Semisimple locally primitive algebras}

To finish our classification of Lie algebras of vector fields in the real plane up to local changes of coordinates, we only have to deal with the semisimple locally primitive algebras. To this end, we shall need a few preliminary definitions and basic results. In the rest of this section, $\mathfrak{g}$ will always denote a locally primitive semisimple Lie algebra of vector fields in $\mathbb{R}^{2}$.

Let

$$
K(X, Y) \equiv \operatorname{tr}(\operatorname{ad} X \cdot \operatorname{ad} Y)
$$

denote the Killing-Cartan bilinear form on $\mathfrak{g}$, where ad $X: \mathfrak{g} \rightarrow \mathrm{g}$ is defined as

$$
\text { ad } X=[X, \cdot] \text {. }
$$

It is well known [10] that $g$ is semisimple if and only if $K$ is non-degenerate, that is,

$$
K(X, Y)=0 \text { for all } Y \in \mathrm{g} \Leftrightarrow X=0 .
$$

Lemma 5.1. For every generic point $P$ we have

$$
\mathrm{g}_{P}^{3}=\{0\}
$$

Proof. If $A \in \mathfrak{g}$ and $B \in \mathfrak{g}_{P}^{3}$, we have from (3.17) that

$$
\operatorname{ad} B \cdot g_{P}^{j} \subset g_{P}^{j+2},
$$

and therefore, applying (3.17) again, that

$$
(\operatorname{ad} A \cdot \operatorname{ad} B) \cdot \mathrm{g}_{P}^{j} \subset \mathrm{g}_{P}^{j+1} .
$$

Now, since

$$
\mathrm{g}=\bigoplus_{j \geqslant 0} \mathrm{~g}_{P}^{j} / \mathrm{g}_{P}^{j+1}
$$

we can construct a basis of $g$ by adding the bases for each of the linear subspaces 
$\mathrm{g}_{p}^{j} / \mathrm{g}_{P}^{j+1}$. Working in such a basis, it is clear that (5.6) implies that

$$
\operatorname{tr}(\operatorname{ad} A \cdot \operatorname{ad} B)=0 \text {, }
$$

or equivalently

$$
K(\mathrm{~g}, B)=0 .
$$

The lemma then follows from the non-degeneracy of $K$.

Proposition 5.2. $\mathrm{g}_{P}^{2}$ is abelian at every generic point $P$.

Proof. The proof is again a simple consequence of (3.17). Indeed,

$$
\left[\mathfrak{g}_{P}^{2}, \mathfrak{g}_{P}^{2}\right] \subset \mathrm{g}_{P}^{3}=\{0\},
$$

where the last equality follows from the previous lemma.

Proposition 5.3. If $P$ is a generic point for $\mathrm{g}$, we have

$$
\operatorname{dim} \mathrm{g}_{P}^{2} \leqslant 2 \text {. }
$$

Proof. The orthogonal complement $\mathrm{g}_{P}^{2, \perp}$ of $\mathrm{g}_{P}^{2}$ with respect to $K$ is given by

$$
\mathrm{g}_{P}^{2, \perp}=\left\{X \in \mathrm{g} \mid K\left(X, \mathrm{~g}_{P}^{2}\right)=0\right\} .
$$

By an argument analogous to that of Lemma 5.1, it is easy to show that

$$
\mathrm{g}_{P} \subset \mathrm{g}_{P}^{2, \perp} .
$$

Since $K$ is non-degenerate, we have

whence

$$
\operatorname{dim} g=\operatorname{dim} g_{P}^{2}+\operatorname{dim} g_{P}^{2, \perp},
$$

$$
\begin{aligned}
\operatorname{dim} \mathfrak{g}_{P}^{2} & =\operatorname{dim} \mathrm{g}-\operatorname{dim} \mathfrak{g}_{P}^{2 . \perp} \\
& \leqslant \operatorname{dim} \mathrm{g}-\operatorname{dim} \mathrm{g}_{P} \\
& =\operatorname{dim} \mathrm{g} / \mathrm{g}_{P}=\operatorname{dim} T_{P} \mathbb{R}^{2}=2
\end{aligned}
$$

The above results put very stringent restrictions on the dimension of $g$. Indeed, from (5.4), it follows that

$$
\begin{aligned}
\operatorname{dim} \mathrm{g} & =\operatorname{dim} \mathrm{g} / \mathrm{g}_{P}+\operatorname{dim} \mathrm{g}_{P} / \mathrm{g}_{P}^{2}+\operatorname{dim} \mathrm{g}_{P}^{2} \\
& =2+\operatorname{dim} \mathfrak{g}_{P} / \mathrm{g}_{P}^{2}+\operatorname{dim} \mathrm{g}_{P}^{2} .
\end{aligned}
$$

But Theorem 3.6 implies that

$$
1 \leqslant \operatorname{dim} g_{P} / g_{P}^{2} \leqslant 2,
$$

when $\mathrm{g}$ is not equivalent to an algebra in the list (3.1); combining this with (5.11) and (5.16) yields the following bounds for $\operatorname{dim} \mathrm{g}$ :

$$
3 \leqslant \operatorname{dim} g \leqslant 6 .
$$

Upon complexification, we obtain

$$
3 \leqslant \operatorname{dim}_{\mathbb{C}} g^{\mathbb{C}} \leqslant 6 .
$$


If $g$ is semisimple, then so will be its complexification $\mathrm{g}^{\mathbb{C}}$; from this and the well-known classification of abstract complex semisimple Lie algebras [10], it follows that either

or

$$
\mathrm{g}^{\mathbb{C}} \approx A_{1} \approx \mathfrak{g l}(2, \mathbb{C})
$$

$$
\mathrm{g}^{\mathbb{C}} \approx A_{1} \oplus A_{1} \approx \mathfrak{g o}(4, \mathbb{C}) \text {. }
$$

Since $A_{1}$ has only two real forms, namely $\mathfrak{s l}(2, \mathbb{R})$ and $\mathfrak{s} \mathfrak{o}(3, \mathbb{P})(\approx \mathfrak{s} \mathfrak{u}(2))$, combining the previous results we obtain:

THEOREM 5.4. If $\mathrm{g}$ is a semisimple locally primitive Lie algebra of vector fields in $\mathbb{R}^{2}$ not equivalent to one of the algebras (3.1), then we have the following alternatives:

$$
\begin{aligned}
& g \approx \mathfrak{S l}(2, \mathbb{R}) \\
& g \approx \mathfrak{S} \mathfrak{o}(3, \mathbb{R}) ; \\
& g^{\mathbb{C}} \approx \mathfrak{S} \mathfrak{o}(4, \mathbb{C}) \quad\left(\Rightarrow \operatorname{dim}_{\mathbb{R}} g=6\right) .
\end{aligned}
$$

In the first two cases,

$$
\mathrm{g}_{P}^{2}=\{0\}
$$

at every generic point $P$, whereas in the third case $g_{P}^{2}$ is abelian and

$$
\operatorname{dim} \mathrm{g}_{P}^{2}=2 .
$$

In the next sections, we shall determine all the equivalence classes of semisimple locally primitive Lie algebras of vector fields in $\mathbb{R}^{2}$ through a case-by-case analysis of the three possibilities (5.22)-(5.24).

\section{Locally primitive algebras isomorphic to $\mathfrak{g l}(2, \mathbb{R})$}

We shall determine in this section all the equivalence classes of semisimple locally primitive algebras of vector fields in the real plane isomorphic to $\mathfrak{I l}(2, \mathbb{R})$. To begin with, let $\left\{X_{0}, X_{+}, X_{-}\right\}$be a basis of $g$ satisfying the standard $\operatorname{sl}(2, \mathbb{R})$ commutation relations

$$
\left[X_{0}, X_{ \pm}\right]= \pm X_{ \pm}, \quad\left[X_{+}, X_{-}\right]=-2 X_{0} .
$$

Let us choose local coordinates $(x, y)$ such that

$$
X_{-}=p
$$

in a neighbourhood of $(0,0)$. If we impose the condition $\left[X_{0}, X_{-}\right]=-X_{-}$, it immediately follows that $X_{0}$ must be of the form

$$
X_{0}=(x+\xi(y)) p+\eta(y) q
$$

The latter expression can be simplified without altering (6.2) by means of a change of variables of the form

$$
\left\{\begin{array}{l}
\tilde{x}=x+\varphi(y) \\
\tilde{y}=\psi(y)
\end{array}\right.
$$


Indeed, if $\varphi(y)$ and $\psi(y)$ solve the ordinary differential equations

$$
\left\{\begin{array}{l}
\eta \psi^{\prime}=\psi \\
\eta \varphi^{\prime}=\varphi-\xi
\end{array}\right.
$$

then (6.2) and (6.3) are transformed into

$$
X_{-}=\tilde{p}, \quad X_{0}=\tilde{x} \tilde{p}+\tilde{y} \tilde{q} .
$$

Therefore we may set

$$
\xi=0, \quad \eta=y
$$

by relabelling the variables $x$ and $y$. Imposing now the condition $\left[X_{+}, X_{-}\right]=$ $-2 X_{0}$, we readily obtain

$$
X_{+}=\left(x^{2} p+2 x y q\right)+\gamma(y) p+\beta(y) q .
$$

Finally, using the remaining commutation relation in (6.1) we arrive at the following system of ordinary differential equations in the unknown functions $\beta(y)$ and $\gamma(y)$ :

whence

$$
y \beta^{\prime}-2 \beta=y \gamma^{\prime}-2 \gamma=0,
$$

$$
\beta=2 c_{1} y^{2}, \quad \gamma=c_{2} y^{2}
$$

Thus we have

$$
X_{+}=\left(x^{2}+c_{2} y^{2}\right) p+2 y\left(x+c_{1} y\right) q
$$

A final change of variables

$$
\bar{x}=x+c_{1} y, \quad \tilde{y}=\left|c_{1}^{2}+c_{2}\right|^{\frac{1}{2}} y
$$

changes (6.11) into

$$
X_{+}=\left(\tilde{x}^{2} \pm \tilde{y}^{2}\right) \tilde{p}+2 \tilde{x} \tilde{y} \tilde{q}
$$

without affecting $X_{0}$ or $X_{-}$. This proves that there are at most two equivalence classes of locally primitive Lie algebras of vector fields in $\mathbb{R}^{2}$ isomorphic to $\mathfrak{I l}(2, \mathbb{R})$, spanned by the vector fields

or

$$
p, x p+y q,\left(x^{2}-y^{2}\right) p+2 x y q
$$

$$
p, x p+y q,\left(x^{2}+y^{2}\right) p+2 x y q .
$$

Of these two Lie algebras of vector fields, only the first one is locally primitive, as is easily verified using Proposition 2.5. Indeed, it is trivial to check that for both (6.14) and (6.15) all points are generic except those on the $x$-axis. A trivial calculation shows that the isotropy subalgebra of (6.14)-(6.15) at a generic point $P=(a, b)(b \neq 0)$ is generated by the vector field

$$
\frac{1}{2 b}\left(X_{+}-2 a X_{0}+X_{-}\right)= \pm \eta p+\xi q
$$

where the ' + ' sign corresponds to (6.15), and $\xi=x-a, \eta=y-b$. According to $\S 2$, the action of (6.16) on $T_{P} \mathbb{R}^{2}$ is given by the matrix

$$
\left(\begin{array}{cc}
0 & \pm 1 \\
1 & 0
\end{array}\right) .
$$


From this and Proposition 2.5 our assertion clearly follows. Thus we have proved the following:

THEOREM 6.1. There is, up to local diffeomorphisms, exactly one equivalence class of locally primitive Lie algebras of vector fields in $\mathbb{R}^{2}$ isomorphic to $\mathfrak{I} l(2, \mathbb{R})$. $A$ representative of this equivalence class is the Lie algebra given by

$$
\operatorname{span}\left\{p, x p+y q,\left(x^{2}-y^{2}\right) p+2 x y q\right\} \text {. }
$$

\section{Locally primitive algebras isomorphic to $\mathfrak{S} 0(3, \mathbb{P})$}

We shall show in this section that there is exactly one equivalence class of locally primitive Lie algebras of vector fields in $\mathbb{P}^{2}$ isomorphic to $\mathfrak{S D}(3, \mathbb{R})$. In addition, we shall find a representative of this equivalence class whose expression is particularly simple in appropriate local coordinates.

We start by choosing a basis $\left\{X_{1}, X_{2}, X_{3}\right\}$ of $g$ obeying the standard $\mathfrak{B o}(3, \mathbb{R})$ commutation relations, namely

$$
\left[X_{1}, X_{2}\right]=X_{3}, \quad\left[X_{2}, X_{3}\right]=X_{1}, \quad\left[X_{3}, X_{1}\right]=X_{2} .
$$

As before, we choose local coordinates $(\theta, r)$ such that, for instance,

$$
X_{1}=\partial_{\theta}
$$

in some open set. It is then easy to see that the commutation relations of $X_{1}$ with $X_{2}$ and $X_{3}$ imply that the latter vector fields are of the form

with

$$
\begin{aligned}
& X_{2}=\cos \theta A(r)+\sin \theta B(r), \\
& X_{3}=-\sin \theta A(r)+\cos \theta B(r),
\end{aligned}
$$

$$
\begin{aligned}
& A(r)=a_{1}(r) \partial_{\theta}+a_{2}(r) \partial_{r}, \\
& B(r)=b_{1}(r) \partial_{\theta}+b_{2}(r) \partial_{r} .
\end{aligned}
$$

We can simplify the form of the vector fields (7.3) without changing $X_{1}$ by performing a change of coordinates of the type

$$
\Theta=\theta+\varphi(r), \quad R=f(r) .
$$

It is readily checked that, under such a change, the vector fields $A$ and $B$ are transformed into

$A(R)=\left[\left(a_{1}+\varphi^{\prime} a_{2}\right) \cos \varphi-\left(b_{1}+\varphi^{\prime} b_{2}\right) \sin \varphi\right] \partial_{\Theta}+\left(a_{2} \cos \varphi-b_{2} \sin \varphi\right) f^{\prime} \partial_{R}$,

$B(R)=\left[\left(a_{1}+\varphi^{\prime} a_{2}\right) \sin \varphi+\left(b_{1}+\varphi^{\prime} b_{2}\right) \cos \varphi\right] \partial_{\Theta}+\left(a_{2} \sin \varphi+b_{2} \cos \varphi\right) f^{\prime} \partial_{R}$.

We can always choose the arbitrary function $\varphi(r)$ in (7.5) so that the coefficient of $\partial_{R}$ in $B$ vanishes. Going back to the old coordinates $(\theta, r)$, we see that this amounts to setting

$$
b_{2}=0
$$

in (7.4). Computing now the Lie bracket of $X_{2}$ with $X_{3}$ we obtain

$$
\left[X_{2}, X_{3}\right]=\left(a_{2} b_{1}^{\prime}-a_{1}^{2}-b_{1}^{2}\right) \partial_{\theta}-a_{1} a_{2} \partial_{r},
$$

and equating it to $X_{1} \equiv \partial_{\theta}$ we finally arrive at the equations

$$
\begin{aligned}
& a_{1}=0, \\
& a_{2} b_{1}^{\prime}=1+b_{1}^{2} .
\end{aligned}
$$


Rescaling the radial coordinate $r$, we can set, without loss of generality,

$$
a_{2}(r)=\frac{1}{2}\left(1+r^{2}\right) \text {. }
$$

With this choice of $a_{2}$, the complete solution of (7.9) is

$$
b_{1}(r)=\frac{1+2 c r-r^{2}}{c\left(1-r^{2}\right)-2 r} \text { with } c \in \mathbb{R}, \quad \text { or } \quad b_{1}(r)=\frac{2 r}{1-r^{2}},
$$

the singular solution corresponding to $c=\infty$. Finally, let us choose $f$ in equation (7.5) as

$$
f(r)=\frac{r-\gamma}{1+\gamma r} \text { or } f(r)=\frac{r-1}{1+r}
$$

where, in the first case, $\gamma$ is any of the two (real) roots of the equation

$$
c \gamma^{2}-2 \gamma-c=0 \text {. }
$$

It is then straightforward to check that $X_{2}$ and $X_{3}$ are transformed into

$$
\begin{aligned}
& X_{2}=\frac{1}{2}\left(1+R^{2}\right) \cos \theta \partial_{R}+\frac{1}{2}\left(R-R^{-1}\right) \sin \theta \partial_{\theta}, \\
& X_{3}=-\frac{1}{2}\left(1+R^{2}\right) \sin \theta \partial_{R}+\frac{1}{2}\left(R-R^{-1}\right) \cos \theta \partial_{\theta} .
\end{aligned}
$$

We thus see that there is exactly one equivalence class of locally primitive Lie algebras of vector fields in the real plane isomorphic to $\mathfrak{S O}(3, \mathbb{R})$, whose basis elements are given by (7.2)-(7.14) in appropriate local coordinates $(\theta, R)$. A simpler expression for the latter generators is obtained by changing back to cartesian coordinates

$$
x=R \cos \theta, \quad y=R \sin \theta ;
$$

namely, up to irrelevant constant factors,

$$
\begin{aligned}
& X_{1}=x q-y p \\
& X_{2}=\left(1+x^{2}-y^{2}\right) p+2 x y q, \\
& X_{3}=2 x y p+\left(1+y^{2}-x^{2}\right) q .
\end{aligned}
$$

This proves the following:

THEOREM 7.1. All locally primitive Lie algebras of vector fields in $\mathbb{B}^{2}$ isomorphic to $\mathfrak{S O}(3, \mathbb{R})$ are equivalent to the Lie algebra spanned by the vector fields (7.15) under a local change of coordinates.

Note that the vector fields (7.15) have the following simple interpretation: the Lie algebra $\mathfrak{S O}(3, \mathbb{R})$ acts on the unit sphere $S^{2} \subset \mathbb{R}^{3}$ by infinitesimal rotations, with generators

$$
\begin{aligned}
& Y_{1}=x \partial_{y}-y \partial_{x} \\
& Y_{2}=z \partial_{x}-x \partial_{z} \\
& Y_{3}=y \partial_{z}-z \partial_{y}
\end{aligned}
$$

The vector fields $X_{1}, X_{2}, X_{3}$ are just the images of $Y_{1}, Y_{2}, Y_{3}$ under the standard stereographic projection from the north pole $(0,0,1)$. 


\section{Locally primitive algebras with complexification isomorphic to $\mathfrak{B D}(4, \mathbb{C})$}

The last case to examine in order to complete our classification of Lie algebras of vector fields in $\mathbb{R}^{2}$ under changes of local coordinates is given by (5.24), namely that of the six-dimensional algebras whose complexification is isomorphic to the complex Lie algebra $\mathfrak{S} \mathfrak{D}(4, \mathbb{C})$.

From the discussion in $\S 5$, we know that in this case $\mathrm{g}_{P}^{2}$ is a two-dimensional abelian subalgebra of $g$, for every generic point $P$. We claim that this implies that there is a generic point $Q \neq P$ such that

$$
\operatorname{dim} \mathrm{g}_{P}^{2}(Q)=2 .
$$

Indeed, if (8.1) is not satisfied at any generic point $Q \neq P$, then there must be a generic point $P^{\prime} \neq P$ such that $g_{P}^{2}(Q)$ is one-dimensional for all $Q$ in some neighbourhood of $P^{\prime}$. If this is the case, the fact that $g_{P}^{2}$ is abelian further implies that we can choose local coordinates around $P^{\prime}$ such that $\mathrm{g}_{P}^{2}$ is generated by the vector fields

$$
X_{1}=p, \quad X_{2}=y p
$$

in a neighbourhood of $P^{\prime}$. It follows that, if $Q \equiv(a, b)$ is any generic point in a sufficiently small neighbourhood of $P^{\prime}$, the isotropy algebra $g_{Q}$ contains the linear vector field

$$
X_{2}-b X_{1}=(y-b) p .
$$

But the action of this vector field on $T_{Q} \mathbb{R}^{2}$ is reducible, since it is given by the matrix

$$
\left(\begin{array}{ll}
0 & 1 \\
0 & 0
\end{array}\right)
$$

hence $g$ must be locally equivalent to the Lie algebra ( $3.1 \mathrm{~b}$ ) by Theorem 3.6 , which is clearly impossible since the latter algebra is not semisimple.

Assume, therefore, that (8.1) holds. Since $\mathrm{g}_{P}^{2}$ is abelian, this implies that there are local coordinates $(x, y)$ around the generic point $Q \neq P$ such that

$$
\mathrm{g}_{P}^{2}=\operatorname{span}\{p, q\}
$$

in a neighbourhood of $Q$, whose coordinates can be taken as $(0,0)$. From Theorem 3.6 and Lemma 5.1, it follows that $g$ is generated in a neighbourhood of $(0,0)$ by the vector fields

$$
p, q, D, R, Q_{1}, Q_{2}
$$

where

$$
\begin{aligned}
& D=x p+y q+\tilde{D} \equiv D_{0}+\tilde{D}, \\
& R=y p-x q+\tilde{R} \equiv R_{0}+\tilde{R}, \\
& Q_{i} \equiv Q_{i 0}+\tilde{Q}_{i}, \quad \text { for } i=1,2 .
\end{aligned}
$$

In the previous formula, the vector fields $\tilde{D}, \tilde{R}$ and $\tilde{Q}_{i}$ are of the following orders at $(0,0)$ :

$$
\begin{aligned}
& \tilde{D}=O\left((|x|+|y|)^{2}\right), \\
& \tilde{R}=O\left((|x|+|y|)^{2}\right), \\
& \tilde{Q}_{i}=O\left((|x|+|y|)^{3}\right),
\end{aligned}
$$


and $Q_{i 0}$ is a quadratic vector field, that is,

$$
Q_{i 0}=\left(a_{i} x^{2}+2 b_{i} x y+c_{i} y^{2}\right) p+\left(d_{i} x^{2}+2 e_{i} x y+f_{i} y^{2}\right) q, \quad \text { for } i=1,2,
$$

with $a_{i}, \ldots, f_{i}$ constant. Moreover, from Proposition 5.2 we know that

$$
\left[Q_{1}, Q_{2}\right]=0 \text {. }
$$

At this point, it is convenient to introduce some complex notation. We let

$$
z=x+i y, \quad \bar{z}=x-i y,
$$

and we define the corresponding partial differential operators

$$
\partial_{z}=\frac{1}{2}(p-i q), \quad \partial_{\bar{z}}=\frac{1}{2}(p+i q) .
$$

Furthermore, let us introduce the complex vector fields

$$
\begin{aligned}
& \mathscr{D}=\frac{1}{2}(D+i R), \\
& \mathscr{Q}=\frac{1}{2}\left(Q_{1}+i Q_{2}\right)
\end{aligned}
$$

and, similarly,

$$
\begin{aligned}
& \mathscr{D}_{0}=\frac{1}{2}\left(D_{0}+i R_{0}\right)=z \partial_{z}, \\
& \mathscr{Q}_{0}=\frac{1}{2}\left(Q_{10}+i Q_{20}\right) .
\end{aligned}
$$

The Lie brackets of the vector fields (8.5) are completely determined by those of their complex counterparts

$$
\partial_{z}, \partial_{\bar{z}}, \mathscr{D}, \bar{D}, \mathscr{Q}, \overline{\mathscr{Q}} \text {, }
$$

and conversely. It follows that the vector fields (8.14) are the generators of a complex Lie algebra, which is nothing but the complexification of $\mathrm{g}$.

Let us now derive the commutation relations of the (complex) Lie algebra spanned by the vector fields (8.14). In the first place, taking into account (3.17), (8.7) and (8.13), we readily obtain:

$$
\begin{aligned}
& {\left[\partial_{z}, \mathscr{D}\right]=\partial_{z}+\sigma_{1} \mathscr{D}+\tilde{\sigma}_{1} \overline{\mathscr{D}}+\mu_{1} \mathscr{Q}+\tilde{\mu}_{1} \overline{\mathscr{Q}}} \\
& {\left[\partial_{\bar{z}}, \mathscr{D}\right]=\sigma_{2} \mathscr{D}+\tilde{\sigma}_{2} \overline{\mathscr{D}}+\mu_{2} \mathscr{Q}+\tilde{\mu}_{2} \overline{\mathscr{Q}}} \\
& {\left[\partial_{z}, \mathscr{Q}\right]=\sigma_{3} \mathscr{D}+\tilde{\sigma}_{3} \overline{\mathscr{D}}+\mu_{3} \mathscr{Q}+\tilde{\mu}_{3} \overline{\mathscr{Q}}} \\
& {\left[\partial_{\bar{z}}, \mathscr{Q}\right]=\sigma_{4} \mathscr{D}+\tilde{\sigma}_{4} \overline{\mathscr{D}}+\mu_{4} \mathscr{Q}+\tilde{\mu}_{4} \overline{\mathscr{Q}},}
\end{aligned}
$$

where $\sigma_{i}, \tilde{\sigma}_{i}, \mu_{i}, \tilde{\mu}_{i}$ are complex constants. From (8.17) and (8.18), upon equating terms of order 1 at $(0,0)$ we obtain

$$
\begin{aligned}
& {\left[\partial_{z}, \mathscr{Q}_{0}\right]=\sigma_{3} \mathscr{D}_{0}+\widetilde{\sigma}_{3} \overline{\mathscr{D}}_{0} \equiv \sigma_{3} z \partial_{z}+\bar{\sigma}_{3} \bar{z} \partial_{\bar{z}},} \\
& {\left[\partial_{\bar{z}}, \mathscr{Q}_{0}\right]=\sigma_{4} \mathscr{D}_{0}+\tilde{\sigma}_{4} \overline{\mathscr{D}}_{0} \equiv \sigma_{4} z \partial_{z}+\bar{\sigma}_{4} \bar{z} \partial_{\bar{z}} .}
\end{aligned}
$$

But, from the Jacobi identity,

$$
\left[\partial_{z},\left[\partial_{\bar{z}}, \mathscr{Q}_{0}\right]\right]=\left[\partial_{\bar{z}},\left[\partial_{z}, \mathscr{Q}_{0}\right]\right]
$$

and the latter formulae, we readily infer that

$$
\tilde{\sigma}_{3}=\sigma_{4}=0 \text {. }
$$

We thus have

$$
\left[\partial_{z}, \mathscr{Q}_{0}\right]=\sigma_{3} z \partial_{z}, \quad\left[\partial_{\bar{z}}, \mathscr{Q}_{0}\right]=\tilde{\sigma}_{4} \bar{z} \partial_{\bar{z}} .
$$


Taking into account (cf. (8.8)) the fact that

$$
\mathscr{Q}_{0}(0)=0
$$

and integrating (8.22), we easily obtain

$$
2_{0}=\frac{1}{2}\left(\sigma_{3} z^{2} \partial_{z}+\bar{\sigma}_{4} \bar{z}^{2} \partial_{\bar{z}}\right)
$$

Replacing the generators 2 and $\overline{\mathscr{Q}}$ by their linear combination

$$
\frac{\bar{\sigma}_{3} 2-\tilde{\sigma}_{4} \overline{2}}{\left|\sigma_{3}\right|^{2}-\left|\bar{\sigma}_{4}\right|^{2}}
$$

and its complex conjugate-which amounts to replacing the generators $Q_{1}$ and $Q_{2}$ of $g$ by a linear combination thereof-we can henceforth assume, without loss of generality, that

$$
2_{0}=\frac{1}{2} z^{2} \partial_{z} \text {. }
$$

(Notice that $\left|\sigma_{3}\right|^{2}-\left|\widetilde{\sigma}_{4}\right|^{2} \neq 0$, since otherwise 2 and $\bar{Q}$, and hence $Q_{1}$ and $Q_{2}$, would be linearly dependent.) Using (8.26), we see that it is straightforward to obtain

$$
\begin{aligned}
& {\left[\partial_{z}, \mathscr{Q}\right]=\mathscr{D}+\mu_{3} \mathscr{Q}+\tilde{\mu}_{3} \overline{\mathscr{Q}}} \\
& {\left[\partial_{\bar{z}}, \mathscr{2}\right]=\mu_{4} \mathscr{Q}+\tilde{\mu}_{4} \overline{\mathscr{Q}}} \\
& {[\mathscr{D}, \mathscr{2}]=\mathscr{Q}} \\
& {[\overline{\mathscr{D}}, \mathscr{Q}]=0}
\end{aligned}
$$

and, from (8.9),

$$
[\mathscr{Q}, \bar{Q}]=0 \text {. }
$$

Adding to $\mathscr{D}$ an appropriate linear combination of $\mathscr{2}$ and $\overline{\mathscr{Q}}$ (which, again, is equivalent to adding to $D$ and $R$ certain linear combinations of $Q_{1}$ and $Q_{2}$ ) we easily check that we can replace the commutation relations (8.15) and (8.16) by the following simpler ones:

$$
\begin{aligned}
& {\left[\partial_{z}, \mathscr{D}\right]=\partial_{z}+\tilde{\sigma}_{1} \overline{\mathscr{D}}+\mu_{1} \mathscr{Q}+\tilde{\mu}_{1} \overline{\mathscr{Q}},} \\
& {\left[\partial_{\bar{z}}, \mathscr{D}\right]=\sigma_{2} \mathscr{D}+\mu_{2} \mathscr{Q}+\tilde{\mu}_{2} \overline{\mathscr{Q}} .}
\end{aligned}
$$

Applying the Jacobi identity

we immediately obtain

$$
\left[\partial_{\bar{z}},\left[\partial_{z}, \mathscr{D}\right]\right]=\left[\partial_{z},\left[\partial_{\bar{z}}, \mathscr{D}\right]\right],
$$

$$
\tilde{\sigma}_{1}=\sigma_{2}=0,
$$

and hence (8.32) further simplifies to

$$
\begin{aligned}
& {\left[\partial_{z}, \mathscr{D}\right]=\partial_{z}+\mu_{1} \mathscr{Q}+\tilde{\mu}_{1} \overline{\mathscr{Q}},} \\
& {\left[\partial_{\bar{z}}, \mathscr{D}\right]=\mu_{2} \mathscr{Q}+\bar{\mu}_{2} \overline{\mathscr{Q}} .}
\end{aligned}
$$

On the other hand, from the first equation in (8.13) it follows that

$$
[\mathscr{D}, \overline{\mathscr{D}}]=\mu_{5} \mathscr{Q}+\tilde{\mu}_{5} \overline{\mathscr{Q}},
$$


where $\mu_{5}$ and $\tilde{\mu}_{5}$ are complex constants. Making use of the Jacobi identity

$$
\left[\partial_{z},[\mathscr{D}, \overline{\mathscr{D}}]\right]=\left[\mathscr{D},\left[\partial_{z}, \overline{\mathscr{D}}\right]\right]+\left[\overline{\mathscr{D}},\left[\mathscr{D}, \partial_{z}\right]\right]
$$

and of equations (8.27)-(8.30) and (8.32), we are led to

$$
\mu_{5}=0 \text {. }
$$

Taking the complex conjugate of (8.36) we then obtain

$$
\bar{\mu}_{5}=-\bar{\mu}_{5}=0 \text {, }
$$

that is,

$$
[\mathscr{D}, \overline{\mathscr{D}}]=0 .
$$

Using once more the Jacobi identity and (8.29)-(8.31), we see that

$$
\begin{aligned}
{\left[\mathscr{Q},\left[\partial_{z}, \mathscr{D}\right]\right] } & =\left[\mathscr{Q}, \partial_{z}\right] \\
& =\left[\partial_{z},[\mathscr{Q}, \mathscr{D}]\right]+\left[\mathscr{D},\left[\partial_{z}, \mathscr{Q}\right]\right] \\
& =\left[\mathscr{Q}, \partial_{z}\right]+\mu_{3} \mathscr{Q},
\end{aligned}
$$

from which we obtain

$$
\mu_{3}=0 \text {. }
$$

Similarly, taking the Lie bracket of $\overline{\mathscr{Q}}$ with $\left[\partial_{\bar{z}}, \mathscr{D}\right]$ we obtain

$$
\tilde{\mu}_{3}=0 \text {, }
$$

that is,

$$
\left[\partial_{z}, \mathscr{Q}\right]=\mathscr{D} \text {. }
$$

In a completely analogous way, computing the Lie bracket $\left[\overline{\mathscr{Q}},\left[\partial_{z}, \mathscr{D}\right]\right]$ we arrive at

$$
\left[\partial_{\bar{z}}, 2\right]=0 \text {. }
$$

Finally, the computation of $\left[\overline{\mathscr{D}},\left[\partial_{z}, \mathscr{D}\right]\right]$ leads to

$$
\tilde{\mu}_{1}=\tilde{\mu}_{2}=0 \text {, }
$$

from which it follows that

$$
\mu_{2}=0
$$

by applying the Jacobi identity to $\left[\partial_{z},\left[\partial_{\bar{z}}, \mathscr{D}\right]\right]$.

For ease of reference, we shall summarize below the commutation relations of the Lie algebra $g^{\mathbb{C}}$ which we have just computed:

$$
\begin{aligned}
& {\left[\partial_{z}, \mathscr{D}\right]=\partial_{z}+\omega^{2} \mathscr{Q},} \\
& {\left[\partial_{z}, \mathscr{2}\right]=\mathscr{D}} \\
& {\left[\partial_{\bar{z}}, \mathscr{2}\right]=\left[\partial_{\bar{z}}, \mathscr{D}\right]=0,} \\
& {[\mathscr{D}, \mathscr{Q}]=\mathscr{Q},} \\
& {[\mathscr{D}, \overline{\mathscr{D}}]=[\mathscr{D}, \overline{\mathscr{Q}}]=[\mathscr{Q}, \overline{\mathscr{Q}}]=0 .}
\end{aligned}
$$

In the latter formulae, we have set

$$
\omega^{2} \equiv \mu_{1}
$$


and we have omitted the commutation relations obtained from the above by complex conjugation, such as

$$
\left[\partial_{\bar{z}}, \bar{D}\right]=\partial_{\bar{z}}+\bar{\omega}^{2} \bar{Q} \text {. }
$$

Equations (8.45)-(8.47), as well as the initial conditions

$$
\mathscr{D}(0)=\mathscr{Q}(0)=0
$$

(cf. (8.6)-(8.8)), completely determine the vector fields $\mathscr{D}$ and 2 . In fact, integrating (8.45) $-(8.47)$ with (8.51) taken into account, one immediately obtains

$$
\mathscr{D}=\omega^{-1} \sinh (\omega z) \partial_{z}, \quad \mathscr{Q}=\omega^{-2}[\cosh (\omega z)-1] \partial_{z},
$$

if $\omega \neq 0$, or

$$
\mathscr{D}=z \partial_{z}, \quad \mathscr{Q}=\frac{1}{2} z^{2} \partial_{z}
$$

if $\omega=0$.

The Lie algebra spanned by the real and imaginary parts of (8.52a) together with the translations $p$ and $q$ is actually equivalent, under a local change of coordinates in $\mathbb{R}^{2}$, to the algebra spanned by $p, q$, and the real and imaginary parts of $(8.52 b)$. Indeed, let us perform the local change of variables

$$
\begin{aligned}
& u=e^{a x-b y} \cos (b x+a y), \\
& v=e^{a x-b y} \sin (b x+a y),
\end{aligned}
$$

with $\omega \equiv a+i b$. In complex notation, (8.53) is simply

$$
w \equiv u+i v=e^{\omega z},
$$

so that we have

$$
\begin{aligned}
& \partial_{z}=\omega e^{\omega z} \partial_{w}=\omega w \partial_{w}, \\
& \mathscr{D}=\frac{1}{2 \omega}\left(e^{\omega z}-e^{-\omega z}\right) \partial_{z}=\frac{1}{2}\left(w^{2}-1\right) \partial_{w}, \\
& \mathscr{Q}=\frac{1}{2 \omega^{2}}\left(e^{\omega z}+e^{-\omega z}-2\right) \partial_{z}=\frac{1}{2 \omega}\left(w^{2}-2 w+1\right) \partial_{w} .
\end{aligned}
$$

Thus, the complex change of variables (8.54) maps the vector fields $\partial_{z}$ and (8.52a) into linear combinations of the vector fields

$$
\partial_{w}, w \partial_{w}, w^{2} \partial_{w}
$$

It thus follows that the change of variables (8.53) will transform the Lie algebra generated by the real and imaginary parts of $\partial_{z}$ and the vector fields (8.52a) into the Lie algebra generated by the real and imaginary parts of (8.56).

Checking that the latter Lie algebra is indeed locally primitive offers no difficulty, thanks again to Proposition 2.5. Thus we have shown that all locally primitive Lie algebras of vector fields in $\mathbb{R}^{2}$ whose complexification is isomorphic to $\mathfrak{s}(4, \mathbb{C})$ are equivalent (under local changes of coordinates) to the Lie algebra generated by the real and imaginary parts of the vector fields (8.56). Using (8.10) and (8.11), we find the following explicit formulae for the generators of the latter Lie algebra:

$$
p, q, x p+y q, y p-x q,\left(x^{2}-y^{2}\right) p+2 x y q, 2 x y p+\left(y^{2}-x^{2}\right) q .
$$


The commutation relations of (8.57) can be easily extracted from (8.45) (with $\omega=0$ ). In fact [23], it is well known that the algebra generated by the vector fields $(8.57)$ is isomorphic to $\mathfrak{S p}(3,1)$, the Lie algebra of the Lorentz group of four-dimensional Minkowski space. Equivalently, it can be shown that (8.57) generates $\mathfrak{I} \mathfrak{I}(2, \mathbb{C})$, when regarded as a Lie algebra over $\mathbb{R}$. Note that the vector fields (8.57) generate the restricted conformal algebra in $\mathbb{P}^{2}$, that is, the corresponding group action is generated by translations, rotations, dilatations and inversions. It is the two-dimensional analogue of the full conformal group in three or more dimensions. (Of course, the full conformal group in the plane $\mathbb{R}^{2} \cong \mathbb{C}$ is infinite-dimensional, since any analytic function $f: \mathbb{C} \rightarrow \mathbb{C}$ gives a conformal transformation away from its critical points.)

As before, we shall finish by summarizing the main results obtained in this section in the following theorem:

THEOREM 8.1. Every locally primitive Lie algebra of vector fields in $\mathbb{R}^{2}$ whose complexification is isomorphic to $\mathfrak{S} \mathfrak{D}(4, \mathbb{C})$ is equivalent to the Lie algebra generated by the vector fields (8.57) under a local change of coordinates. In particular, all such algebras are isomorphic to $\mathfrak{B O}(3,1)$.

Interestingly, $\mathfrak{I O}(3,1)$ is the only real form of $\mathfrak{g} \mathfrak{o}(4, \mathbb{C})$ which can be realized by a primitive Lie algebra of real vector fields in the plane. On the other hand, $\mathfrak{S o}(2,2)$ is the only real form of $\mathfrak{g} \mathfrak{D}(4, \mathbb{C})$ which can be realized by an imprimitive Lie algebra of real vector fields in the plane. There is a direct geometrical interpretation of this result. The representation of $\mathfrak{s} \mathfrak{v}(2,2)$ given in entry 16 of Table 1 reflects the well-known Lie algebra isomorphisms

$$
\mathfrak{S} \mathfrak{o}(2,2) \cong \mathfrak{I} \mathfrak{l}(2, \mathbb{R}) \oplus \mathfrak{s l}(2, \mathbb{R}) \cong \mathfrak{S} \mathfrak{o}(2,1) \oplus \mathfrak{b} \mathfrak{o}(2,1) .
$$

The quotients of the corresponding Lie group $O(2,1)$ by its subgroups come into play now. In standard notation, $\mathfrak{S 0}(2,1)$ is spanned by the infinitesimal hyperbolic rotations $K_{i}$ around the $x^{i}$-axes $(i=1,2)$ and the infinitesimal rotation $L_{3}$ around the $x^{3}$-axis. The quotient $O(2,1) / G(1)$, where $G(1)$ is the connected subgroup generated by $K_{2}+L_{3}$, is isomorphic to the upper sheet of a cone (cf. [21]). The action of $O(2,1)$ on this cone has an invariant foliation provided by the generators of the cone. The action becomes primitive on the one-dimensional quotient $O(2,1) / G(2)$, where $G(2)$ is the connected subgroup generated by $\left\{K_{1}, K_{2}+L_{3}\right\}$, isomorphic to the affine group on the line. (Alternatively, one could form the quotient $O(2,1) / O(1,1)$, which is isomorphic to a one-sheeted hyperboloid [21]. The $O(2,1)$ action has two invariant foliations given by the rulings of the hyperboloid.)

Thus we see that the imprimitive action of $\mathfrak{g} \mathfrak{D}(2,2)$ on $\mathbb{P}^{2}$ depends crucially on the decomposition (8.58). None of the other real forms $g$ of $\mathfrak{s o}(4, \mathbb{C})$ enjoy decompositions of the form $g=g_{1} \oplus g_{2}$, such that the action of the corresponding subgroup $G_{i}$ on the two-dimensional homogeneous space $G_{i} / H_{i}$, where $H_{i} \subset G_{i}$ is a closed subgroup, admits an invariant foliation. For example, for the real form

$$
\mathfrak{S} \mathfrak{o}(4, \mathbb{R})^{*} \cong \mathfrak{S} \mathfrak{o}(2,1) \oplus \mathfrak{S} \mathfrak{o}(3, \mathbb{R}),
$$

we see that $\operatorname{SO}(3, \mathbb{R})$ has no invariant foliation on $S^{2} \cong \operatorname{SO}(3, \mathbb{R}) / \operatorname{SO}(2, \mathbb{R})$, and such a decomposition would be needed for $\mathfrak{S D}(4, \mathbb{R})^{*}$ to act imprimitively on $\mathbb{R}^{2}$. Similar arguments hold for the other real forms of $\mathfrak{B o}(4, \mathbb{C})$. 


\section{Concluding remarks}

In the previous sections, we have completely classified all (real analytic) finite-dimensional Lie algebras of vector fields in the real plane under changes of local coordinates. Apart from the algebras found by Lie in the complex case, we have found five additional equivalence classes, namely the Lie algebras (4.1), (4.2), (6.14), (7.15) and (8.57). The complete list of equivalence classes of finite-dimensional Lie algebras of vector fields in $\mathbb{R}^{2}$ under local changes of variables is therefore as shown in Table 1 (p. 341).

In Table 1 , we have omitted the trivial algebra $\{0\}$ whose only element is the zero vector field. The functions $1, \xi_{1}(x), \ldots, \xi_{r}(x)$ appearing in Cases 20 and 21 should be linearly independent. The functions $\eta_{1}(x), \ldots, \eta_{r}(x)$ appearing in Cases 22 and 23 are not arbitrary, but must form a basis of solutions for an $r$ th order homogeneous ordinary linear differential equation with constant coefficients. The symbol $\mathfrak{g} \ltimes \mathfrak{h}$ indicates the semi-direct product of the Lie algebras $\mathfrak{g}$ and $\mathfrak{h}$, where $\mathfrak{h}$ is a $g$-module. For instance, $\mathfrak{h}_{2}=\mathbb{R} \ltimes \mathbb{R}$ is the unique solvable two-dimensional Lie algebra.

For the locally primitive algebras, there are two maximal algebras, \#7 $\approx$ $\mathfrak{S o}(3,1)$ and $\# 8 \approx \mathfrak{S}(3, \mathbb{R})$. Note that algebras $\# 1,2,3,4$ are all contained in $\# 7$, and we also have the chains of inclusions

$$
1 \subset 4 \subset 6 \subset 8, \quad 1 \subset 5 \subset 6 \subset 8 \text {. }
$$

As for the structure of these Lie algebras, \#2, 3, 7, 8,11,16,17, 18, are the only semi-simple algebras. The algebras $\# 9,20$ are abelian. The algebras $\# 1,4,10$, $12,13,21,22,23,24,25,26$ are solvable. In Case 10, the nilradical is spanned by $p$; in Cases $1,4,12,13$, it is spanned by $p, q$. In Case 21 , the nilradical is the subalgebra of type 20 . In Case 22 , the nilradical is abelian, spanned by all the vector fields $\eta_{i}(x) q$, unless all the $\eta_{i}$ are polynomials, in which case \#22 is nilpotent, and the vector fields $\eta_{i}(x) q$ span the maximal abelian ideal. Similarly, in Case 23, the nilradical is also spanned by all the vector fields $\eta_{i}(x) q$, unless all the $\eta_{i}$ are of the form $p_{i}(x) e^{\lambda x}$ for a single exponent $\lambda$, in which case the vector field $p+\lambda y q$ is also in the nilradical, which is then no longer abelian. Cases 24,25 and 26 all have non-abelian nilradicals, spanned by $p, q, x q, \ldots, x^{r-1} q$, and, in Cases 24 and 26, also $x^{r} q$. For the remaining cases, which are neither solvable nor semi-simple, the Levi decomposition is either explicitly indicated (Cases 5, 15, 27) or follows immediately from the usual Levi decomposition $\mathfrak{g l}(2)=\mathfrak{g l}(2) \oplus \mathbb{P}$ of the general linear algebra (Cases 6, 14, 19, 28). This completes our short discussion of these Lie algebras.

Finally, we identify some of the particular low-dimensional Lie algebras in our table with those in the general classification scheme of Patera, Sharp, Winternitz and Zassenhaus [20] and Turkowski [22]. The algebra $\mathfrak{h}_{2}$ is the same as $A_{2,1}$ in this classification. Lie algebra \#1 is $A_{3,7}^{\alpha}$ if $\alpha \neq 0$, or $A_{3,6}$ if $\alpha=0$. Algebras \#4, 5, 6 are $A_{4,12}, L_{5,1}$, and $L_{6,3}$, respectively. Finally, algebra $\# 12$ is $A_{3,5}^{\alpha}$ if $\alpha^{2} \neq 1, A_{3,3}$ if $\alpha=1$, or $A_{3,4}$ if $\alpha=-1$. All the other algebras in our list are well-known, or direct sums of well-known ones, or of variable dimension $r$.

\section{Acknowledgement}

We would like to thank Thomas Hawkins for alerting us to some of the historical references. 


\section{References}

1. Y. Alhassid, J. Engel, and J. Wu, 'Algebraic approach to the scattering matrix', Phys. Rev. Lett. 53 (1984) 17-20.

2. L. BIANCHI, Lezioni sulla teoría dei gruppi continui finiti di trasformazioni (Enrico Spoerri, Editore, Pisa, 1918).

3. G. W. Bluman and S. KumeI, Symmetries and differential equations (Springer, New York, 1989).

4. W. BoothBy, 'A transitivity problem from control theory', J. Differential Equations 17 (1975) 296-307.

5. J. E. CAMPBELL, Introductory treatise on Lie's theory of finite continuous transformation groups (The Clarendon Press, Oxford, 1903).

6. É. Cartan, 'Sur la structure des groupes de transformations finis et continus', Thèse, Paris, Nony, $2^{\mathrm{e}}$ edition, Vuibert, 1913; Euvres complètes, part I, vol. 1 (Gauthier-Villars, Paris, 1952), pp. 137-287.

7. M. GolUBITSKy, 'Primitive actions and maximal subgroups of Lie groups', J. Differential Geom. 7 (1972) 175-191.

8. A. González-López, N. Kamran, and P. J. Olver, 'Lie algebras of differential operators in two complex variables', Amer. J. Math., to appear.

9. R. HeRmAnn and M. ACKerman, Sophus Lie's 1880 transformation group paper (Math. Sci. Press, Brookline, Mass., 1975).

10. N. JACOBSON, Lie algebras (Interscience, New York, 1962).

11. N. Kamran and P. J. Olver, 'Equivalence of differential operators', SIAM J. Math. Anal. 20 (1989) 1172-1185.

12. N. KAMRAN and P. J. Olver, 'Lie algebras of differential operators and Lie-algebraic potentials', J. Math. Anal. Appl. 145 (1990) 342-356.

13. R. D. LeVINE, 'Lie algebraic approach to molecular structure and dynamics', Mathematical frontiers in computational chemical physics (ed. D. G. Truhlar), IMA Volumes in Mathematics and its Applications 15 (Springer, New York, 1988), pp. 245-261.

14. S. LIE, 'Gruppenregister', Gesammelte Abhandlungen, vol. 5 (B. G. Teubner, Leipzig, 1924), pp. 767-773.

15. S. LIE, 'Theorie der Transformationsgruppen', Math. Ann. 16 (1880) 441-528; Gesammelte Abhandlungen, vol. 6 (B. G. Teubner, Leipzig, 1927), pp. 1-94. See reference [9] for an English translation.

16. S. LIE, Theorie der Transformationsgruppen, vol. 3 (B. G. Teubner, Leipzig, 1893).

17. P. J. Olver, 'Symmetry groups and group invariant solutions of partial differential equations', $J$. Differential Geom. 14 (1979) 497-542.

18. P. J. Olver, Applications of Lie groups to differential equations (Springer, New York, 1986).

19. R. S. PALAIS, A global formulation of the Lie theory of transformation groups, Memoirs of the American Mathematical Society 22 (Providence, R. I., 1957).

20. J. Patera, R. T. Sharp, P. Winternitz, and H. Zassenhaus, 'Invariants of real low dimension Lie algebras', J. Math. Phys. 17 (1976) 986-994.

21. S. SHNIDER and P. WINTERNITZ, 'Nonlinear equations with superposition principles and the theory of transitive primitive Lie algebras', Lett. Math. Phys. 8 (1984) 69-78.

22. P. Turkowski, 'Low-dimensional real Lie algebras', J. Math. Phys. 29 (1988) 2139-2144.

23. P. WinTERNITZ, 'Comments on superposition rules for nonlinear coupled first-order differential equations', J. Math. Phys. 25 (1984) 2149-2150.

\section{Artemio González-López \\ Depto. Fúsica Teórica II Universidad Complutense 28040 Madrid Spain}

\author{
Niky Kamran \\ Department of Mathematics \\ McGill University \\ Montréal \\ Québec H3A $2 K 6$ \\ Canada
}

\author{
Peter J. Olver \\ School of Mathematics \\ University of Minnesota \\ Minneapolis \\ Minnesota 55455 \\ U.S.A
}

